\title{
A Study of Mach 0.75 Jets and Their Radiated Sound Using Large-Eddy Simulation
}

\author{
Niklas Andersson*, Lars-Erik Eriksson ${ }^{\dagger}$ and Lars Davidson ${ }^{\dagger}$ \\ Chalmers University of Technology, Division of Thermo and Fluid Dynamics, \\ SE-412 96 Göteborg, Sweden
}

\begin{abstract}
Large-Eddy Simulations (LES) of a compressible nozzle/jet configuration have been carried out. Two jets were simulated, an isothermal jet and a jet with a higher temperature than the quiescent surrounding air. The Mach number was in both cases 0.75 and the jet Reynolds number was $5.0 \times 10^{4}$. Sound pressure levels in far-field observer locations were evaluated using Kirchhoff surface integration. The Favre filtered Navier-Stokes equations were solved using a finite volume method solver with a low-dissipation third-order upwind scheme for the convective fluxes, a second-order centered difference approach for the viscous fluxes and a three-stage second-order Runge-Kutta technique in time. The computational domain was discretized using a block structured boundary fitted mesh with approximately $3.0 \times 10^{6}$ cells. The calculations were performed on a parallel computer, using message-passing interface (MPI). A compressible form of Smagorinsky's subgrid scale model was used for computation of the subgrid scale stresses. Absorbing boundary conditions based on characteristic variables were adopted for all free boundaries. Velocity components specified at the entrainment boundaries were estimated from corresponding Reynolds Averaged Navier-Stokes (RANS) calculations, which enable the use of a rather narrow domain. This, furthermore, ensures that the correct amount of fluid is entrained into the domain. Two-point space-time correlations were obtained for locations in the shear layer center, from which length and time scales of turbulence structures were evaluated. Predicted near-field flow statistics and far-field sound pressure levels (SPL) are both in good agreement with experiments. Predicted (SPL) are for all observers locations, where evaluated, within a 3.0 [dB] deviation from measured levels and for most locations within a 1.0 [dB] deviation. Experimental data used for validation were provided by Laboratoire d'Etude Aeródynamiques, Poitiers, France.
\end{abstract}

\section{Nomenclature}

\section{Roman letters}

$c \quad$ speed of sound

$C_{p} \quad$ specific heat at constant pressure

$C_{R}, C_{I}$ Smagorinsky model coefficients

${ }^{*}$ Ph.D. Student, Chalmers University of Technology, Division of Thermo and Fluid Dynamics, Hörsalsvägen 7, SE-412 96 Göteborg, Sweden

†Professor, Chalmers University of Technology, Division of Thermo and Fluid Dynamics, Hörsalsvägen 7, SE-412 96 Göteborg, Sweden

Copyright (c) 2004 by Niklas Andersson, Lars-Erik Eriksson \& Lars Davidson. Published by the American Institute of Aeronautics and Astronautics, Inc. with permission. 
$D_{j} \quad$ nozzle outlet diameter

$e \quad$ energy

$f \quad$ frequency

$k \quad$ kinetic energy

$\mathcal{L} \quad$ integral length scale

$L_{c} \quad$ potential core length

$p \quad$ pressure

$\operatorname{Pr} \quad$ Prandtl number

$q_{j} \quad$ energy diffusion vector

$\mathcal{R} \quad$ correlation amplitude

$r \quad$ radial coordinate or distance from source to observer

$R e_{D} \quad$ Reynolds number based on the jet diameter

$S_{i j} \quad$ strain rate tensor

St Strouhal number $\left(S t=\left(f D_{j}\right) / U_{j}\right)$

$\mathcal{T}$ integral time scale

$T$ temperature

$t \quad$ time

$(u, v, w)$ axial, radial and tangential velocity component

$u_{i} \quad$ cartesian components of velocity vector

$\mathbf{x} \quad$ flow field location

$x_{i} \quad$ cartesian coordinate vector component

y observer location

\section{Greek letters}

$\Delta \quad$ filter width

$\delta_{i j} \quad$ Kronecker delta

$\mu \quad$ dynamic viscosity

$\rho$ density

$\sigma_{i j} \quad$ viscous stress tensor

$\tau \quad$ temporal separation

$\tau_{i j} \quad$ subgrid scale stress tensor

$\tau_{r} \quad$ retarded time

$\theta \quad$ angle from the $x$-axis

$\xi \quad$ spatial separation

Subscripts

0 total condition

$\infty \quad$ free stream or ambient conditions

$j \quad$ jet, nozzle exit condition

$t \quad$ turbulent quantity

\section{Superscripts}

- $\quad$ spatially filtered quantity

, resolved fluctuation

$\sim \quad$ spatially Favre filtered quantity

$\wedge \quad$ Fourier transform

$S G S$ subgrid scale

\section{Symbols}

$\langle\ldots\rangle_{\theta}$ circumferentially averaged quantity

$\langle\ldots\rangle_{t}$ time averaged quantity 


\section{Introduction}

7 HE number of commercial aircraft in service is continuously increasing and airports around the world are growing in size. Moreover, new airports are often built in the vicinity of cities. Restrictions of noise levels in the surroundings of airports have made the reduction of near-ground operation noise an important issue for aircraft and engine manufacturers, and noise generation has now become an important design factor that is taken into consideration early in the construction process. Flowinduced aircraft noise can be divided into two categories: airframe noise and noise generated by the jet engine. The first category includes noise generated by landing gear, high lift devices and the aircraft fuselage itself and the second includes turbo-machinery noise, core noise and jet noise. At take-off, the main sources of noise are the propelling jet and the engine fan, of which the jet exhaust is usually the strongest noise source at full power. In commercial aircraft, increasing the by-pass ratio, i.e. the ratio of air passing through the engine in the by-pass duct to the air passing through the engine core, has given a significant reduction in aircraft noise. However, although increasing the by-pass ratio leads to reduced noise levels, the major motivation for this development has not been noise reduction. Rather, the development towards more efficient engines has led to the use of a higher by-pass ratio with noise reduction as a positive side effect. The lower noise levels of high by-pass ratio (HBR) engines are directly attributable to the reduction in jet noise resulting from lower jet velocities. Unfortunately, without a step change in technology, the maximum by-pass ratio is limited by a number of factors, e.g. the length of the fan blades, rotor speed and engine nacelle drag, and large engines are currently very close to this limit. Consequently, the possibility for reducing jet noise by increasing the by-pass ratio is rapidly decreasing. Other techniques to lower the noise levels have been investigated in the past decades. Among these concepts, many are of a mixing enhancement nature, e.g. lobed mixers, chevrons and tabs. The lobed mixer efficiently evens out velocity differences of the core flow and the by-pass flow, which reduces the exhaust velocity and hence the generated sound. Chevrons and tabs are both devices added to the nozzle geometry that protrude into the flow and thereby enhance the mixing of core, fan and ambient air streams. While these noise-reducing concepts have proven to be able to lower the noise levels, the reduction comes with an efficiency penalty. The contradiction of noise reduction for near-ground operation and requirements for higher thrust and engine efficiency at cruise conditions will probably be common for all new noise-reducing concepts. Furthermore, requirements for higher thrust are often satisfied by increasing the flow through existing engines with only minor modifications, which leads to higher exhaust velocities and temperatures and increases the contribution of the jet to the overall noise.

New noise-reducing concepts will arise from a better understanding of the source mechanisms. For evaluation of the performance of these new concepts, reliable methods for modeling of the source mechanisms must be available. The work presented in this paper has been done within an EU project, $\mathrm{JEAN}^{\mathrm{a}}$, that focused on investigating jet noise both numerically and experimentally. Various known methodologies for noise prediction were tested in the project and compared for a few test cases.

Using a grid fine enough in the far-field regions to minimize the introduction of sound propagation errors, the acoustic field can be obtained directly from the flow field simulation. This requires a detailed numerical compressible flow simulation, e.g. direct numerical simulation (DNS), see for example Freund ${ }^{1}$ and Mitchell et al. ${ }^{2}$ or large-eddy simulation (LES), as done by for example Bogey et al. ${ }^{3}$ and Mankbadi et al. ${ }^{4}$ In DNS, all scales of the turbulent flow field are computed accurately, which requires a mesh fine enough to capture even the smallest scales of the flow, whereas in LES only the large scales of the flow are resolved and the influence on these large scales of the smaller, unresolved scales are modeled using a subgrid scale model. With the computational resources available today, DNS is restricted to fairly simple geometries and low Reynolds number flows. Moreover, it is believed, see Mankbadi, ${ }^{5}$ that large scales are more efficient than small ones in generating sound, which justifies the use of LES for sound predictions. To save computational time, a hybrid approach may be used in

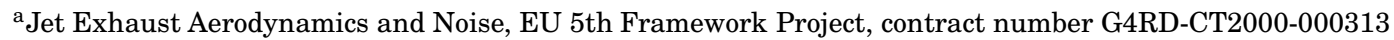


which the computational problem is divided into two parts. An LES can be used to obtain the unsteady non-linear near-field, which in the jet noise case corresponds to the hydrodynamic jet region. Sources of sound obtained from the flow simulation are then propagated to far-field observer locations using, for example, Kirchhoff surface integration, see Andersson et al. ${ }^{6}$ and Andersson. ${ }^{7}$

LES and DNS have been used for jet flow applications in a number of publications. These mostly study jets at moderate Reynolds number due to the high computational costs of performing simulations of a high Reynolds number jet. Many of these studies have been carried out in order to predict jet noise. However, as it is a free shear flow frequently occurring in both nature and industrial applications, the jet is interesting to study in itself. Some studies are thus pure investigations of flow phenomena.

Andersson et al. ${ }^{6}$ used LES and Kirchhoff surface integration for prediction of flow field and radiated sound of a isothermal Mach 0.75 jet. The Reynolds number in this simulation was $\operatorname{Re}_{D}=5.0 \times 10^{4}$. Results were found to be in good agreement with measurements although the Reynolds number was significantly higher in the experiments. In Andersson ${ }^{7}$ heating effects on the jet development and the emitted sound was investigated by comparing a heated jet at Mach number 0.75 with the isothermal jet reported in Ref. (7). The feasibility of using LES for both the flow field and the radiated sound from a high subsonic $6.5 \times 10^{4}$ Reynolds number jet has been discussed by Bogey et al. ${ }^{3,8,9}$ In Refs. $(3,8)$, the acoustic field was obtained directly from the flow simulation. Noise generation mechanisms were found to be relatively independent of Reynolds number. In Ref. (9) Lighthill's acoustic analogy was used in combination with compressible LES to obtain the acoustic field. Bogey \& Bailly ${ }^{10}$ investigated the effects of inflow conditions on the flow field and the radiated sound of a high Reynolds number, $R e_{D}=4.0 \times 10^{5}$, Mach 0.9 jet. Both the flow development and the emitted sound were shown to depend appreciably on initial parameters. The effects of inflow conditions on the self-similar region were studied by Boersma et al. ${ }^{11}$ using DNS. The Reynolds number in this study was $2.4 \times 10^{3}$. Freund ${ }^{1}$ investigated sources of sound in a Mach 0.9 jet at a Reynolds number of $R e_{D}=3.6 \times 10^{3}$ using DNS. In this work the part of the Lighthill source that may radiate to the far-field was isolated using Fourier methods. It was found that the peak of the radiating source coincides with neither the peak of the total source nor the peak of turbulence kinetic energy. The flow field and the radiated sound of a supersonic low Reynolds number jet (Mach $1.92, R e_{D}=2.0 \times 10^{3}$ ) was predicted using DNS by Freund et al. ${ }^{12}$ DeBonis \& Scott ${ }^{13}$ used LES to obtain the flow field of a supersonic high Reynolds number jet (Mach $1.4, \operatorname{Re}_{D}=1.2 \times 10^{6}$ ) from which two-point space-time correlations in the jet shear layer were obtained. Turbulence scales obtained from the correlations were found to be in good agreement with theory. In this study the nozzle geometry was included in the calculation domain. Shur et al. ${ }^{14}$ made simulations of a cold Mach 0.9 jet at a Reynolds number of $1.0 \times 10^{4}$. Radiated sound was successfully predicted using Ffowcs Williams-Hawkings surface integral formulation. The simulation was conducted using only $5.0 \times 10^{5}$ cells and leaving the subgrid-scale model out. Le Ribault et al. ${ }^{15}$ performed large-eddy simulations of a plane jet at two Reynolds numbers, $R e=3.0 \times 10^{3}$ and $R e=3.0 \times 10^{4}$. Simulations were performed for both Reynolds numbers using different subgrid scale models to investigate the ability of the models to capture the jet flow physics. Zhao et al. ${ }^{16}$ conducted an LES of a Mach 0.9 jet at Reynolds number $3.6 \times 10^{3}$ and a jet at Mach 0.4 and a Reynolds number of $5.0 \times 10^{3}$. In this study, radiated sound was obtained both directly from the LES and by using Kirchhoff surface integration. The effect on the radiated sound of the subgrid scale model used was investigated. It was found that using a mixed subgrid-scale model resulted in both higher turbulence levels and sound levels. Rembold et al. ${ }^{17}$ investigated the transition process of a rectangular jet at Mach 0.5 and a Reynolds number of $5.0 \times 10^{3}$ using DNS.

\section{Overview of the Present Study}

Large-eddy simulations of a Mach 0.75 nozzle/jet configuration were performed. The Reynolds number based on the nozzle exit diameter and the jet velocity at the nozzle exit plane, $R e_{D}$, was 
$5.0 \times 10^{4}$. The Reynolds number in the measurements by Jordan et al. ${ }^{18,19}$ and Jordan \& Gervais ${ }^{20}$ used for comparison and validation of the simulations, was approximately one million. Such a high Reynolds number probably means that the scales that needs to be resolved are too small. Thus the Reynolds number in our LES was decreased with the assumption that the flow is only weakly Reynolds number dependent. The nozzle used in the simulation corresponds to the last contraction of the nozzle configuration in the experimental setup.

Simulations of the Mach 0.75 jet were performed for two flow conditions: an unheated jet, i.e. a jet where the static temperature in the nozzle exit plane, $T_{j}$, was equal to the static temperature of the ambient air, $T_{\infty}$, and a heated version where the exhaust temperature was twice that of the surrounding air. The main reason for choosing the Mach 0.75 jet was that experimental data were available for both isothermal and heated conditions. This made it possible to investigate whether heating effects on both the flow field and on radiated sound could be accurately captured with the LES/Kirchhoff approach. Furthermore, both the jet Mach number and the temperature ratio used for the heated jet are quite realistic for real jet engines. Table (1) gives flow properties defining the flow fields of the two jet simulated. Differences are highlighted using bold face numbers. The two jets, i.e. the isothermal and heated Mach 0.75 jet, will hereafter be referred to as Jet I and Jet II, respectively.

Table 1. Flow properties

\begin{tabular}{llll}
\hline \hline & & Jet I & Jet II \\
\hline$U_{j} / c_{\infty}$ & & 0.75 & 0.75 \\
$T_{j} / T_{\infty}$ & & $\mathbf{1 . 0}$ & $\mathbf{2 . 0}$ \\
$P_{\infty}$ & $P a$ & 101300 & 101300 \\
$\rho_{\infty}$ & $\mathrm{kgm}^{-3}$ & 1.225561 & 1.225561 \\
$c_{\infty}$ & $m s^{-1}$ & 340.174 & 340.174 \\
$U_{\infty}$ & $m s^{-1}$ & 0.0 & 0.0 \\
$T_{\infty}$ & $K$ & 288.0 & 288.0 \\
$T_{0_{j}}$ & $K$ & $\mathbf{3 2 0 . 4}$ & $\mathbf{6 0 8 . 4}$ \\
$R e_{D^{*}}$ & & $5.0 \times 10^{4}$ & $5.0 \times 10^{4}$ \\
\hline \hline
\end{tabular}

* The Reynolds number was decreased using modified viscosity

\section{A. Aerodynamic and Acoustic Measurements Used for Validation}

The measurements were made by Jordan et al. ${ }^{18,19,20}$ at the MARTEL facility of CEAT (Centre d'Etudes Aeródynamiques et Thermiques), Poitiers, France. Two-component single-point and monocomponent two-point measurements were made using Laser Doppler Velocimetry (LDV). The acoustic field was sampled using an arc of microphones at 30 jet diameters and 50 jet diameters from the jet exit, respectively. For more detail on the experimental setup see Jordan et al. ${ }^{18,19}$ and Jordan \& Gervais. $^{20}$ 


\section{Governing equations}

The equations solved are the spatially Favre filtered continuity, momentum and energy equations,

$$
\begin{gathered}
\frac{\partial \bar{\rho}}{\partial t}+\frac{\partial\left(\bar{\rho} \tilde{u}_{i}\right)}{\partial x_{i}}=0 \\
\frac{\partial\left(\bar{\rho} \tilde{u}_{i}\right)}{\partial t}+\frac{\partial\left(\bar{\rho} \tilde{u}_{i} \tilde{u}_{j}\right)}{\partial x_{j}}=-\frac{\partial \bar{p}}{\partial x_{i}}+\frac{\partial \bar{\sigma}_{i j}}{\partial x_{j}}+\frac{\partial \tau_{i j}}{\partial x_{j}} \\
\frac{\partial\left(\bar{\rho} \tilde{e}_{0}\right)}{\partial t}+\frac{\partial\left(\bar{\rho} \tilde{e}_{0} \tilde{u}_{j}\right)}{\partial x_{j}}=-\frac{\partial \bar{p} \tilde{u}_{j}}{\partial x_{j}}+ \\
+\frac{\partial}{\partial x_{j}}\left(C_{p} \frac{\mu}{\operatorname{Pr}} \frac{\partial \tilde{T}}{\partial x_{j}}+q_{j}\right)+\frac{\partial}{\partial x_{j}}\left(\tilde{u}_{i}\left(\bar{\sigma}_{i j}+\tau_{i j}\right)\right)
\end{gathered}
$$

where $\bar{\sigma}_{i j}$ and $\tau_{i j}$ are the Favre filtered viscous stress tensor and subgrid scale viscous stress tensor, respectively. These are here defined as

$$
\begin{gathered}
\bar{\sigma}_{i j}=\mu\left(2 \tilde{S}_{i j}-\frac{2}{3} \tilde{S}_{m m} \delta_{i j}\right) \\
\tau_{i j}=\mu_{t}\left(2 \tilde{S}_{i j}-\frac{2}{3} \tilde{S}_{m m} \delta_{i j}\right)-\frac{2}{3} \bar{\rho} k^{S G S} \delta_{i j}
\end{gathered}
$$

where $k^{S G S}$ is the subgrid scale kinetic energy

$$
k^{S G S}=C_{I} \Delta^{2} \tilde{S}_{m n} \tilde{S}_{m n}
$$

$\mu_{t}$ the subgrid scale dynamic viscosity

$$
\mu_{t}=C_{R} \bar{\rho} \Delta^{2} \sqrt{\tilde{S}_{m n} \tilde{S}_{m n}}
$$

and $\tilde{S}_{i j}$ is the Favre filtered strain rate tensor given by,

$$
\tilde{S}_{i j}=\frac{1}{2}\left(\frac{\partial \tilde{u}_{i}}{\partial x_{j}}+\frac{\partial \tilde{u}_{j}}{\partial x_{i}}\right)
$$

The subgrid heat flux appearing in the Favre-filtered energy equation is modeled using a temperature gradient approach

$$
q_{j}=C_{p} \frac{\mu_{t}}{P r_{t}} \frac{\partial \tilde{T}}{\partial x_{j}}
$$

The filter-width used in Eqs. (6) and (7) is the local grid cell width, i.e. $\Delta=\left(\Delta_{1} \Delta_{2} \Delta_{3}\right)^{1 / 3}$. The subgrid scale model used in this work is the Smagorinsky part of the model proposed by Erlebacher et al. ${ }^{21}$ for compressible flows. The constants $C_{R}$ and $C_{I}$ appearing in Eqs. (6) and (7) are the Smagorinsky model constants. These are here given by

$$
\left\{\begin{array}{l}
C_{R}=0.12 \\
C_{I}=0.66
\end{array}\right.
$$

The system of governing equations, Eqs. (1-3), is closed by making assumptions concerning the thermodynamics of the gas considered. It is assumed that the gas is thermally perfect, i.e. it obeys the gas law. Furthermore, the gas is assumed to be calorically perfect, which implies that internal energy and enthalpy are linear functions of temperature. 


\section{Sound Propagation}

Sir James Lighthill's introduction of his acoustic analogy, ${ }^{22}$ i.e. the analogy between the full nonlinear flow and the linear theory of acoustics, is often referred to as the start of aeroacoustics. By combining the time derivative of the continuity equation and the divergence of the momentum equation, the flow equations may be rewritten in a wave operator form. ${ }^{22}$ An analytical solution may be obtained by the use of a free-space Green's function and the sound pressure level in a far-field observer location can be estimated by integration over a volume containing all sound-generating sources. It is important to note that no interaction between the propagating sound wave and the flow field in which it is propagating is considered explicitly, i.e. no refraction or convection effects are taken into account. The equation produced by this pioneering work has later been modified in several ways to include, for example, the effects of the mean-flow acoustic interactions, flow inhomogeneities and surfaces. ${ }^{23}$

\section{A. Kirchhoff Surface Integral Formulation}

Kirchhoff integration is a method for predicting the value of a property, $\Phi$, governed by the wave equation, at a point outside a surface enclosing all generating structures. ${ }^{24}$ The method was originally used in the theory of diffraction of light and in other problems of an electromagnetic nature but has recently been used extensively for aeroacoustic applications. The integral relation is given by

$$
\Phi(\mathbf{y}, t)=\frac{1}{4 \pi} \int_{S}\left[\frac{\Phi}{r^{2}} \frac{\partial r}{\partial n}-\frac{1}{r} \frac{\partial \Phi}{\partial n}+\frac{1}{c_{\infty} r} \frac{\partial r}{\partial n} \frac{\partial \Phi}{\partial t}\right]_{\tau_{r}} d S(\mathbf{x})
$$

where $\mathbf{y}$ is a observer location in the far-field and $\mathbf{x}$ a location on the surface. $\tau_{r}$ denotes that the expression within brackets is to be evaluated at retarded time, i.e. emission time. $\tau_{r}$ is related to the observer evaluation time, $t$, by the distance from the surface to the observer, $r=|\mathbf{y}-\mathbf{x}|$, and the speed of sound in the far-field region, $c_{\infty}$, as

$$
\tau_{r}=t-\frac{r}{c_{\infty}}
$$

The variable, $\Phi$, to be evaluated is in this case the surface pressure. $S$ denotes the surface enclosing all sound generating structures and $n$ denotes the direction normal to the surface. The surface, $S$, must be placed in a region where the flow is completely governed by a homogeneous linear wave equation with constant coefficients. ${ }^{25}$ More detail on the Kirchhoff surface integration method can be found in e.g. Freund et al. ${ }^{25}$ and Lyrintzis. ${ }^{24}$ Since the hydrodynamic source region decays slowly downstream, it is not possible to use a surface enclosing the entire source region without entering this non-linear region, see figure (1). It is thus common practice to use Kirchhoff surfaces that is not closed in the upstream and downstream ends. It was shown by Freund et al. ${ }^{25}$ that the errors introduced by using such surfaces are small if the main portion of the sound sources are within the axial extent of the surface and if lines connecting observer locations with locations in the hydrodynamic region, representing the main sources of sound, intersect with the surface. Rahier et al. ${ }^{26}$ found that the downstream closing surface only gives a minor contribution to the radiated sound. In the work presented in this paper a Kirchhoff surface closed in the upstream end and open in the downstream end was used, see figure (1).

The Kirchhoff surface integral method is less computationally expensive and storage demanding than a method based on volume integrals, e.g. Lighthill's acoustic analogy, since only surface data needs to be stored and evaluated. Moreover, the integrand includes only first-order derivatives, whereas the integrand in Lighthill's acoustic analogy contains second-order derivatives in time or space. One of the main strengths of the Kirchhoff surface integral approach contrary to many other methods is the fact that as long as all sources of sound, flow inhomogeneities and objects that in some way affects the radiated sound is bounded by the surface all these effects are included. This means, for example, that no extra treatment have to be considered to be able to predict the effects of refraction and 


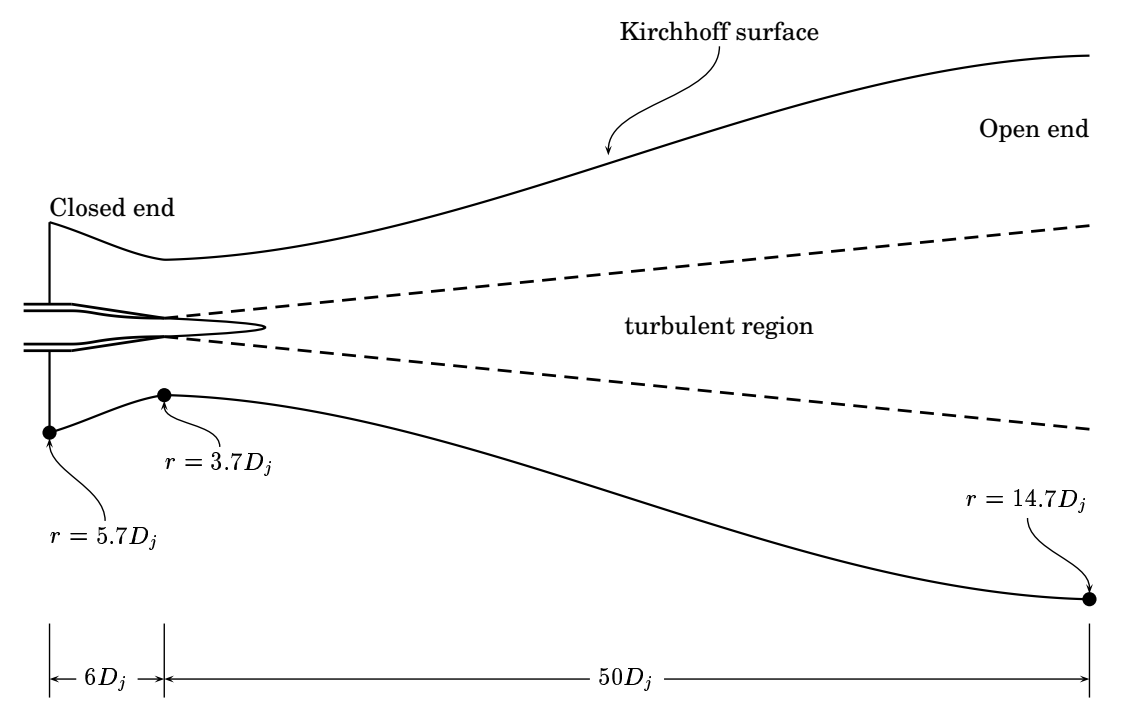

Figure 1. The Kirchhoff surface used is closed in the upstream end and open in the downstream end

convection. This is one of the facts that make this method attractive. Moreover, a strength as well as a weakness of the surface integral approach is the effect of numerical dissipation on the predicted sound. Acoustic waves approaching the integration surface will be low-pass filtered by the numerical method used. A result of this artificial dissipation is that spurious high-frequency noise not supported by the mesh and scheme used will be filtered out and thus not contribute to the predicted far-field sound. This numerical dissipation is not only advantageous since the ability to capture the high-frequency range of the acoustic pressure spectra depends strongly on the mesh resolution in the Kirchhoff surface region. The ideal situation would be to place the integration surface as far as possible from the sources to ensure that all non-linearities appear within the integration surface. The distance from the source to the surface is, however, often limited by the numerical dissipation introduced by the method used. Using Ffowcs William-Hawkings ${ }^{27}$ surface integral method on a porous surface formulation allows the surface to enter regions with weak hydrodynamic fluctuations, which means that the surface can be placed somewhat closer to the sources of sound, see e.g. Shur et al. ${ }^{14}$ and Rahier et al. ${ }^{26}$

\section{Method}

\section{A. Numerical Scheme}

The Favre filtered Navier-Stokes equations were solved using a finite volume method solver with a third-order low-dissipation upwind scheme for the convective fluxes and a centered difference approach for the diffusive fluxes. The temporal derivatives were estimated using a second-order three-stage Runge-Kutta technique. The solver used is based on the G3D family of codes developed by Eriksson. ${ }^{28}$

\section{B. Computational Setup}

Figure (2) gives an overview of the computational domain used for the LES. In this figure, the upper half of the three-dimensional domain used for the calculations is shown. In order to minimize the 
effect of reflections at the domain outlet on the predicted flow field, a damping zone was added at the domain outlet. The functionality of this damping zone or buffer layer has been described in more detail in Andersson et al. ${ }^{6}$ and Andersson. ${ }^{7}$ The axial extent of the physical part of the domain downstream of the nozzle exit is 2.5 meters, which is equal to 50 nozzle diameters $\left(D_{j}=50 \mathrm{~mm}\right)$. The radial extent is $10 D_{j}$ at the nozzle exit plane and $20 D_{j}$ at the domain outlet. The physical part of the computational domain was discretized using a block-structured boundary fitted mesh with 33 mesh blocks and approximately $3 \times 10^{6}$ cells, see figures (3(a)-3(b)). Additionally $5 \times 10^{5}$ cells were used to discretize the damping zone. In order to avoid a center line singularity and to establish mesh homogeneity through out the domain, a combination of polar and cartesian blocks was used, see figure (3(b)). The grid cells are stretched in the downstream direction and radially towards the boundaries using cubic Hermite grid point distribution.

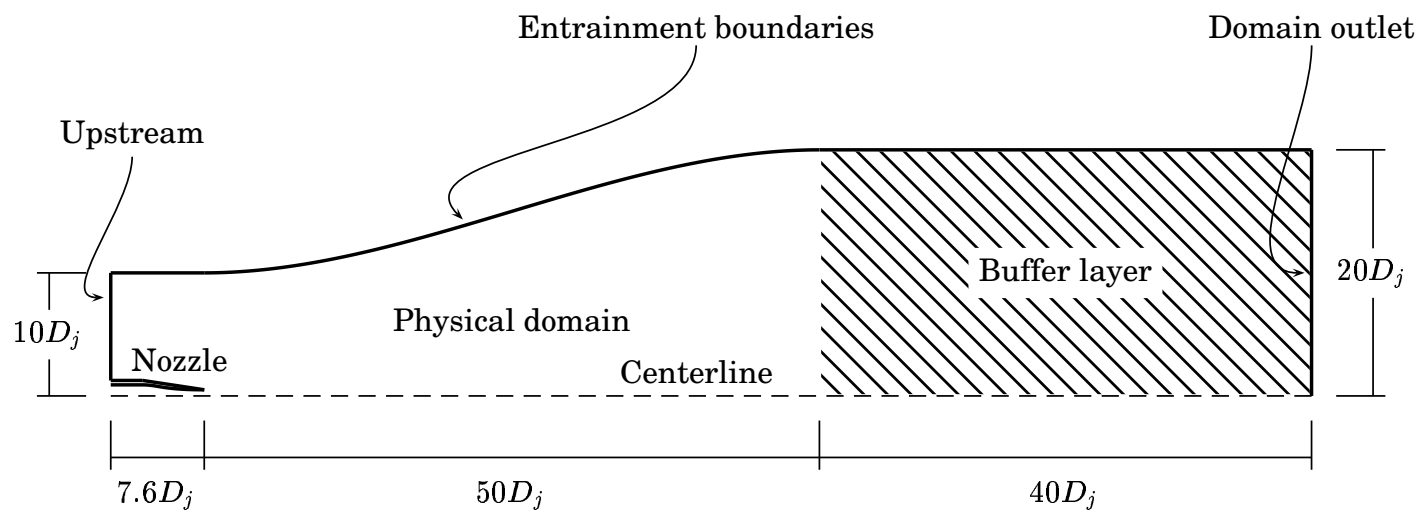

Figure 2. Computational setup

For all free boundaries, i.e. the upstream and entrainment boundaries, absorbing boundary conditions based on the method of characteristics were adopted. Static pressure was specified at the domain outlet, i.e. at the downstream end of the buffer layer, and at the nozzle inlet $P_{0}$ and $h_{0}$ were specified. The velocities specified at the entrainment boundaries were interpolated from corresponding RANS calculations. The calculation domain used for these RANS computations is sufficiently large to ensure that no disturbances caused by the outlet boundaries will reach the location of the LES domain boundaries. Figure (4) shows a comparison of the LES and RANS calculation domains.

\section{Retarded Time}

The concept of retarded time evaluation is shown schematically in figure (5). The upper row of cells in the figure represents the observer pressure signal and the lower rows represent the history of sound sources for four surface elements. Each cell denotes an instant in time and represents a mean of a number of solver time steps. The lowest of these four sources is located farthest away from the observer. This is the source location that will define the starting point of the observer pressure signal. The first observer pressure sample that gets a contribution from the source location farthest away from the observer will be the first complete sample. Cells marked with a black point do not contribute to the observer pressure signal. In the same way, the source location closest to the observer defines the temporal extent of the complete pressure signal since this source will give the last contribution to the signal. The cells filled with gray indicate the time steps for which data have to be stored in the 


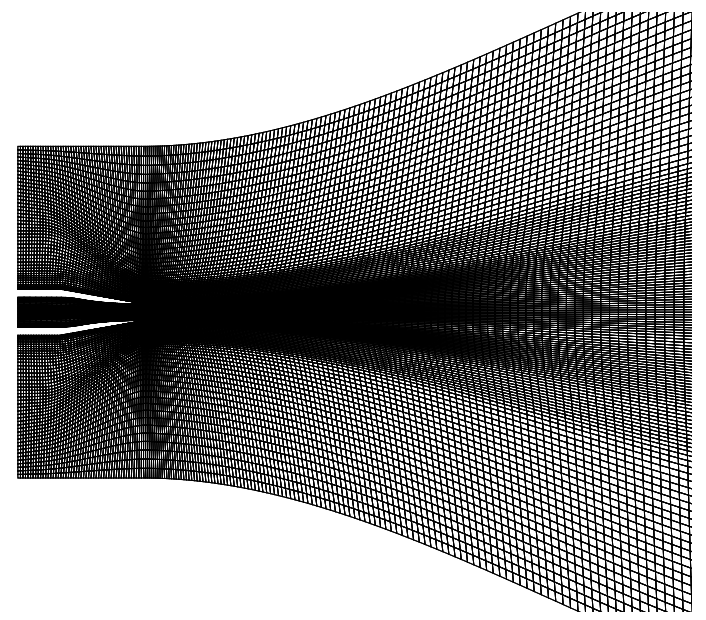

(a) A slice through the calculation domain made at $z=0$, i.e. a $x y$-plane, is depicted. The figure shows the domain inlet including the nozzle and the outer boundaries in the radial direction. The axial extent of the computational domain is roughly twice that shown in the figure.

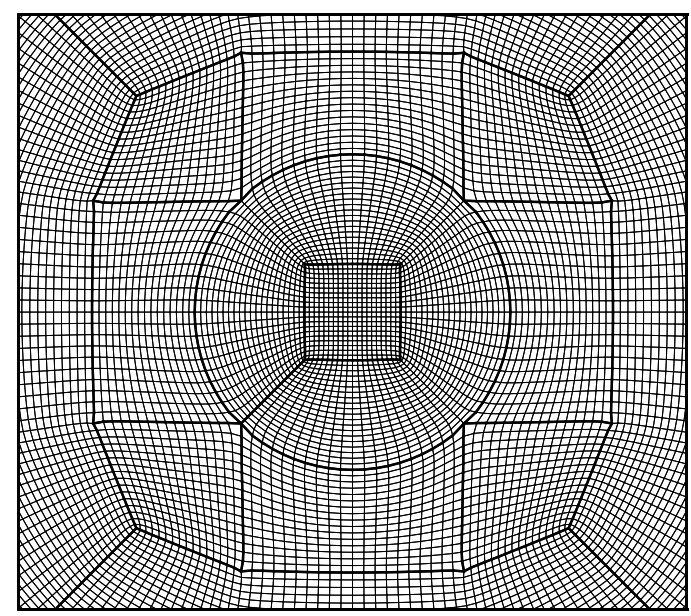

(b) A slice through the calculation domain at constant $x$, i.e. a $y z$-plane, is depicted. Combining cartesian and polar grid blocks enhances the radial direction grid homogeneity throughout the domain.

Figure 3.

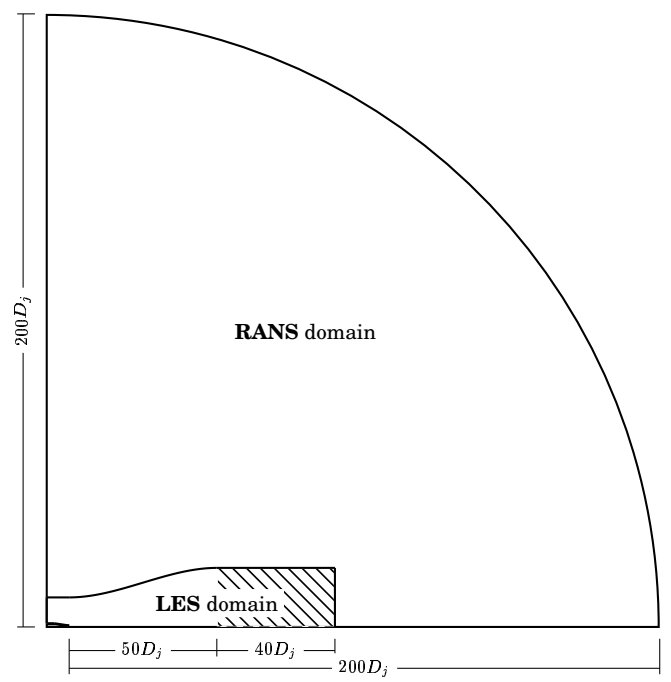

Figure 4. Entrainment velocities were obtained from RANS calculations performed using a significantly larger domain.

solver. Five time steps are needed since fourth-order accurate temporal derivatives are used. This means that the source derivatives are evaluated at two increments in time from the current solver 
time. The observer pressure signal is composed of a number of pressure samples separated in time by a predefined time increment. The arrival time of sound waves is defined by the generation time, speed of sound and the distance to the observer. It is most unlikely that the arrival time will match the predefined discrete instants representing the pressure signal. Thus each surface element contributes to the pressure signal at two discrete times in the observer pressure signal, i.e. the two instants in time closest to the arrival time. The contribution to each of these is obtained by interpolation. The retarded time integration has been implemented such that only the observer pressure signals are stored on disk, which is much less memory consuming than it would be to store the surface data and calculate the pressure signal afterwards.

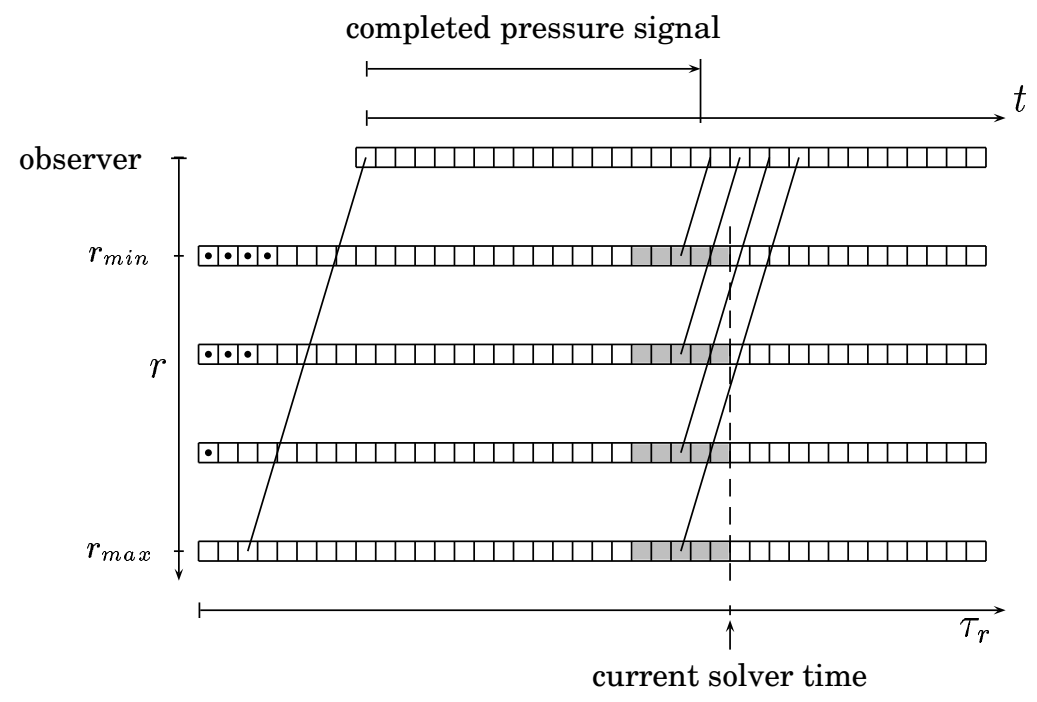

Figure 5. Retarded time

\section{Results}

This section gives predictions of near-field aerodynamics of a Mach 0.75 jet and its radiated sound. The calculations were started up using initial solutions interpolated from 2D RANS calculations. For Jet $I$, the simulation ran for about $1.0 \times 10^{5}$ time steps $(\Delta t=0.25 \mu \mathrm{s})$ before sampling was initiated. This corresponds to 0.025 seconds of simulated time or roughly 3.5 acoustic through-flows, i.e. the time required for an acoustic wave to travel through the calculation domain, not including the outlet buffer layer, based on the speed of sound at ambient conditions. Sampling of statistical data was then continued for another 20 acoustic through-flows. Each acoustic through-flow corresponds to roughly 32 hours of computer wall-time using 14 AMD $1700^{+}$processors of our Linux cluster. For Jet II, as for jet Jet $I$, about 3.5 acoustic through-flows were completed before sampling of data was initiated which in this case corresponds to roughly $1.7 \times 10^{5}$ time steps, $(\Delta t=0.15 \mu \mathrm{s})$. Sampling of statistical data was then continued for about 14 acoustic through-flows. In this case, due to the shorter time step, each acoustic through-flow corresponds to roughly 54 hours of computer wall time. The time steps were chosen such that the CFL number was not larger than 0.5 anywhere.

$$
C F L=\frac{(|u|+c) \Delta t}{\Delta x}
$$

Profiles of statistical quantities to be presented in the following sections are obtained from flow field data that were averaged in both time and the azimuthal direction to establish improved statistical

11 of 24 
convergence. The profiles are made non-dimensional using the jet velocity at the nozzle exit, $U_{j}$, and the nozzle outlet diameter, $D_{j}$. More detail on the results presented in this section can be found in Andersson ${ }^{7}$ and Andersson et al. ${ }^{6}$

\section{A. Instantaneous and Time-averaged Flow Field}

Figure (6) depicts contours of instantaneous axial velocity in a plane along the jet axis, $y=0$. Comparing the contours in figure (6(a)), which represent Jet I, with those obtained for Jet II, see figure (6(b)), It seems that heating the jet results in a higher initial mixing and hence a shorter potential core region. Furthermore, the heated jet seems to be narrower than the isothermal jet. According to Hinze, ${ }^{29}$ experiments have indicated that increasing the temperature ratio $T_{j} / T_{\infty}$ gives a narrower jet. Furthermore, it has been observed that the length of the potential core decreases with increased temperature ratio. ${ }^{29}$ Figure (7) shows contours of instantaneous axial velocity in a plane cutting through the jet at $x=3.0 D_{j}$. These figures clearly visualize the asymmetric nature of the jet at an instant in time.

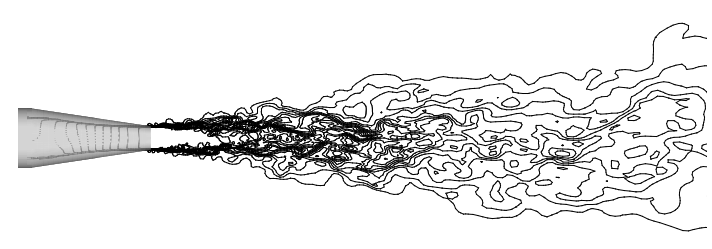

(a) Jet I

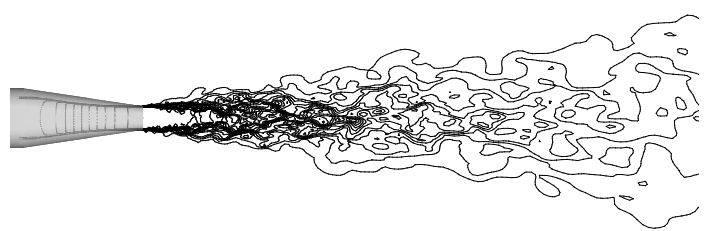

(b) Jet II

Figure 6. Contours of instantaneous axial velocity $(y=0)$

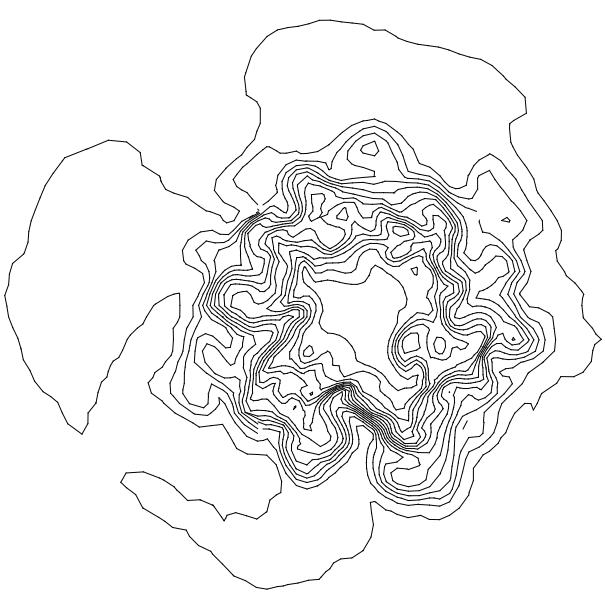

(a) Jet I

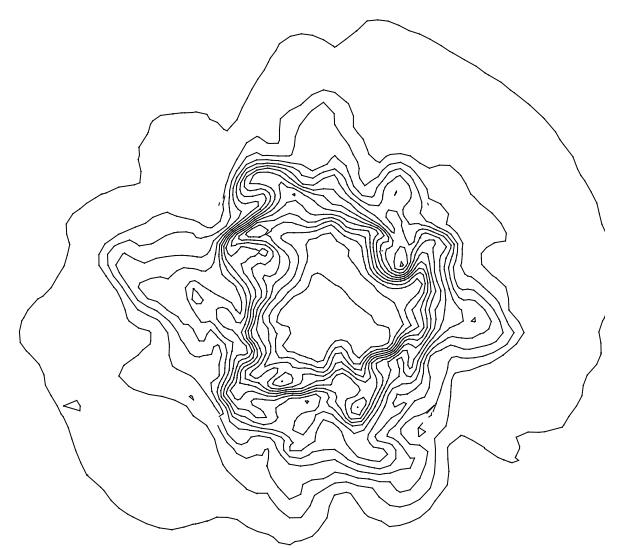

(b) Jet II

Figure 7. Contours of instantaneous axial velocity $\left(x=3.0 D_{j}\right)$ 
Profiles of mean flow quantities were extracted along the centerline and along radial lines at three axial positions downstream of the nozzle exit according to figure (8). Although the time averaged flow field is generally in good agreement with the measured quantities, there are some discrepancies. In the predicted flow field the initial mixing is overpredicted, resulting in a shorter potential core region. Figure (9) gives a comparison of predicted and measured centerline axial velocity for the two jets. Defining the length of the potential core, $L_{c}$, as the axial location where the averaged axial velocity is $0.95 U_{j}$ gives for Jet I $L_{c}=5.5 D_{j}$ and for Jet II $L_{c}=4.0 D_{j}$, which should be compared with $L_{c}=$ $6.5 D_{j}$ and $L_{c}=5.0 D_{j}$ for the experimental data. Figure (10) shows radial profiles of axial velocity. Figure (11) gives a comparison of turbulence intensities. The differences in levels of $u_{r m s}$ and $v_{r m s}$ indicates that the turbulence anisotropy is captured. The peak levels of predicted turbulence intensity are shifted towards the nozzle exit, which is consistent with the underprediction of potential core lengths since the maximum turbulence intensity is found where the potential core closes. Figure (12) shows radial profiles of $u v$ correlations. The maximum correlation levels are overpredicted near the nozzle exit, which again is consistent with the higher degree of mixing found in the LES data.

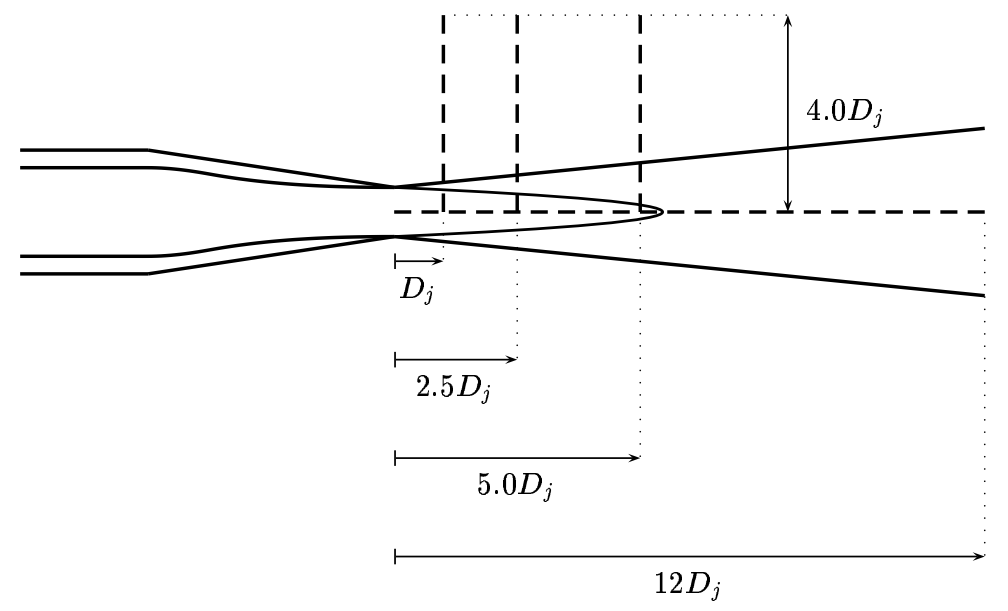

Figure 8. The dashed lines denotes lines along which profiles of time-averaged quantities were extracted

\section{B. Two-point Space-time Correlations}

Two-point space-time correlations were obtained for the axial velocity component in a few shear layer locations along the nozzle lip line. The two-point space-time correlation of axial velocity for a certain spatial separation, $\xi$, and separation in time, $\tau$, is given by

$$
\mathcal{R}_{u u}(\mathbf{x}, \xi, \tau)=\frac{\left\langle u^{\prime}(\mathbf{x}, t) u^{\prime}(\mathbf{x}+\xi, t+\tau)\right\rangle_{t}}{\sqrt{\left\langle u^{\prime 2}(\mathbf{x})\right\rangle_{t}} \sqrt{\left\langle u^{\prime 2}(\mathbf{x}+\xi)\right\rangle_{t}}}
$$

where $u^{\prime}$ denotes resolved fluctuation of axial velocity and $\mathbf{x}$ is the position in the flow field where the two-point correlation is evaluated, see figure (13). Figure (14) shows correlations obtained in the shear layer at an axial location corresponding to the potential core closure. The curve centered at $\tau=0$ corresponds to the autocorrelation and each consecutive curve is obtained by increasing the spatial separation, $\xi$, by $0.3 D_{j}$. For homogeneous isotropic turbulence the correlation peak would be unity for all spatial separations, i.e. the increasing deviation from unity with increasing spatial separation is a measure of how far from frozen the turbulence is. The shape of the correlation curves as well as the 


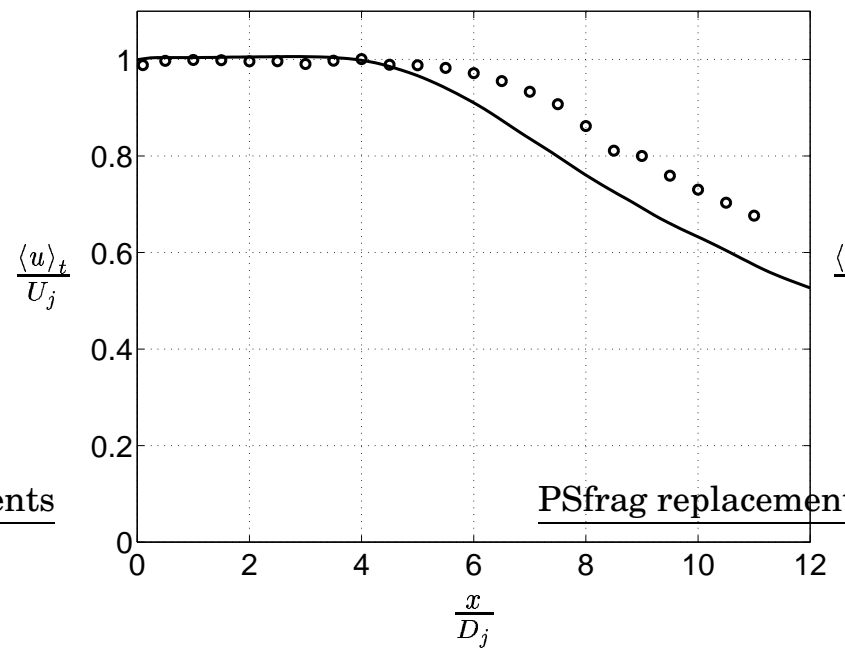

(a) Jet I

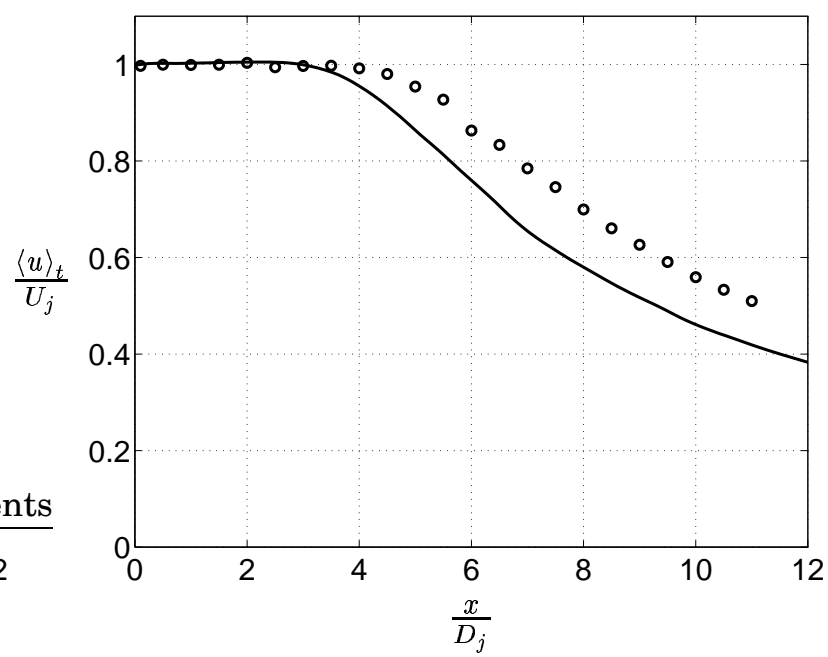

(b) Jet II

Figure 9. Centerline profile of time-averaged axial velocity. Solid line denotes LES and circles measurements. ${ }^{18}$

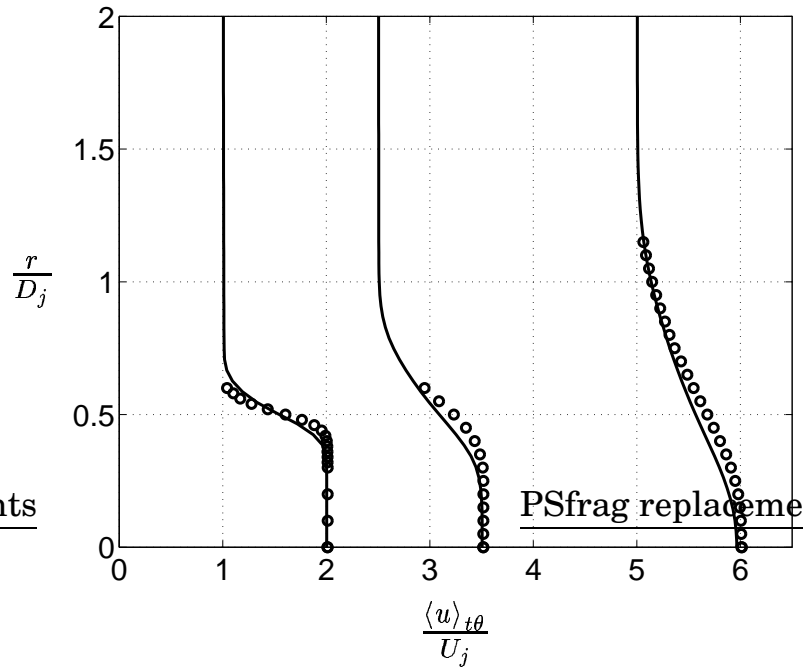

(a) Jet I

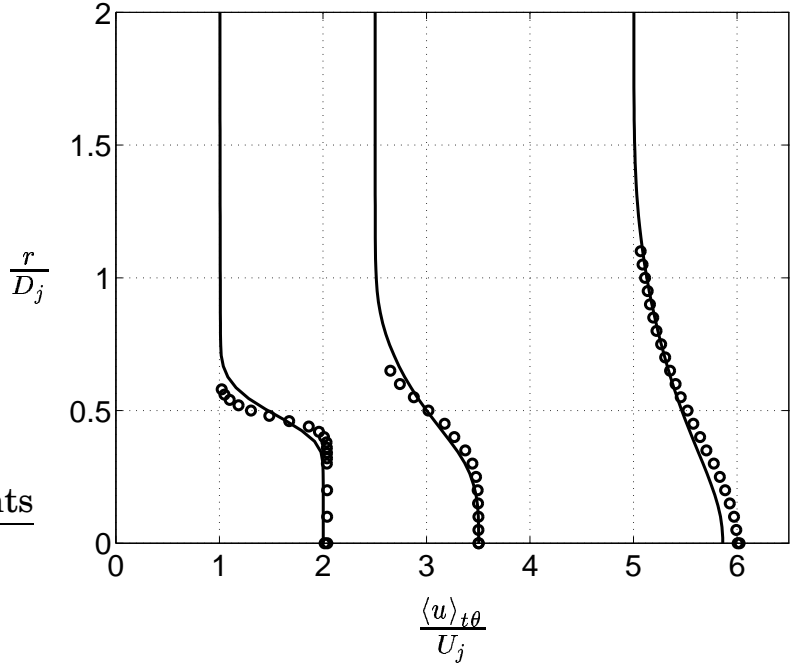

(b) Jet II

Figure 10. Radial profiles of axial velocity. The profiles have been staggered according to their axial location. See also legend to figure (9). 


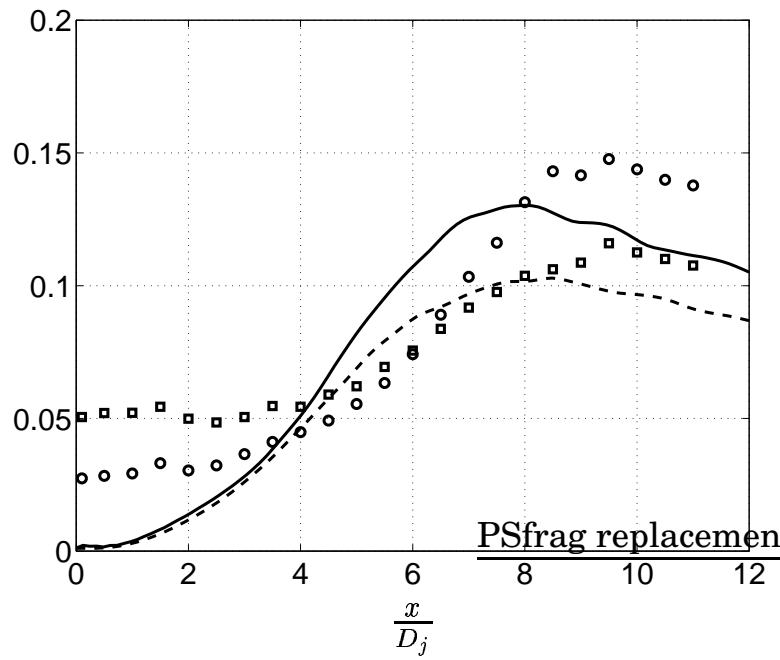

(a) Jet I

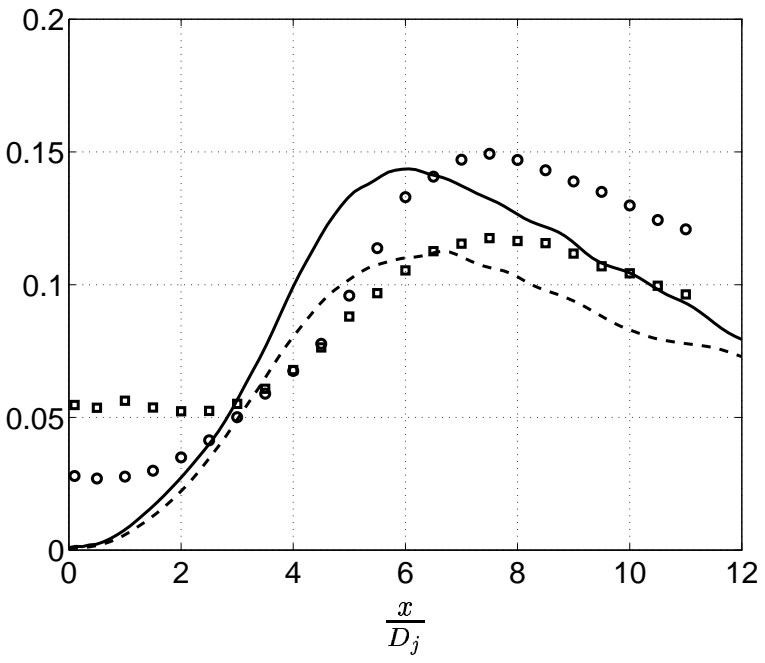

(b) Jet II

Figure 11. Axial profiles of turbulence intensities. solid line and dashed line denotes $u_{r m s}$ and $v_{r m s}$, respectively. Circles and squares denotes the corresponding measured ${ }^{18}$ quantities.

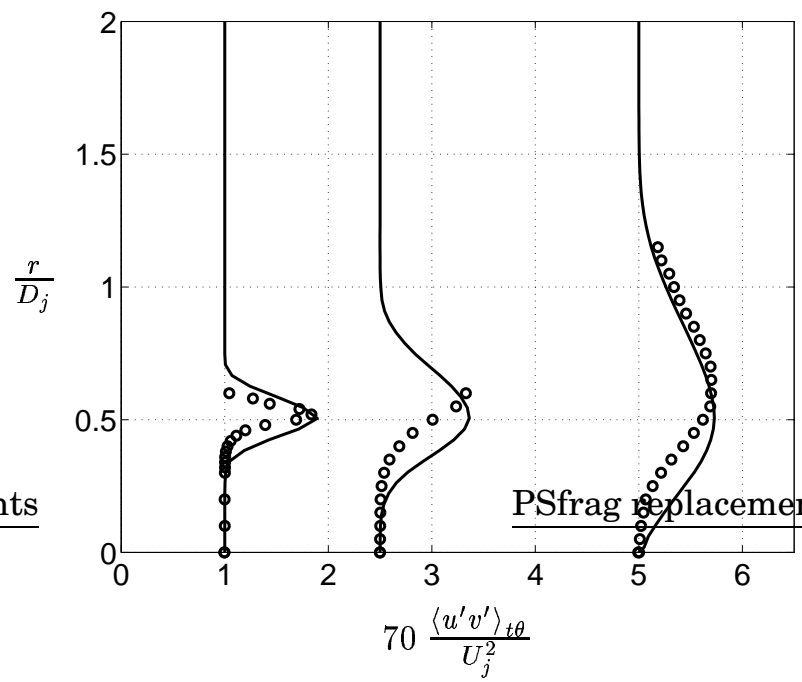

(a) Jet I

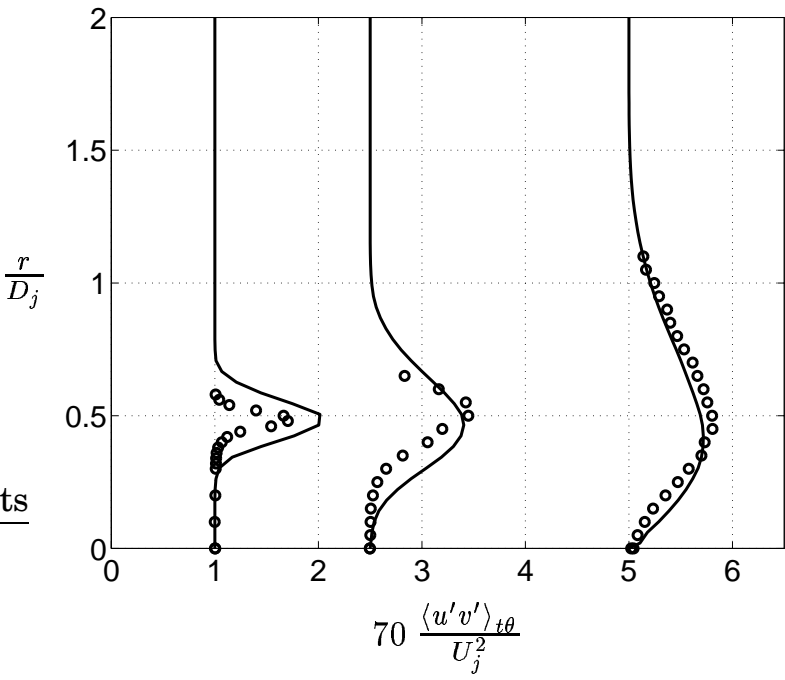

(b) Jet II

Figure 12. Radial profiles of predicted and measured $u v$ correlation. See also legend to figure (9). 


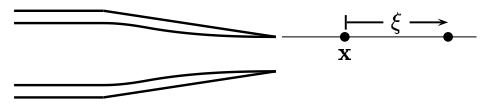

Figure 13. Two-point space-time correlations were evaluated in shear layer locations along the nozzle lip line, $x$. The spatial separation, $\xi$, is downstream in the axial direction.

correlation envelope, i.e. the curve connecting the correlation peaks, is in rather good agreement with the measured data. Figure (15) depicts contours of two-point space-time correlations in the $\xi \tau$-plane. Again, the correlations have been obtained in the shear layer at the end of the potential core. The peaks of the predicted correlations are indicated by stars and those of the correlations obtained from the two-point measurements ${ }^{20, b}$ are indicated by circles. The slope of a line connecting the correlation peaks corresponds to the local convection velocity of turbulence structures.

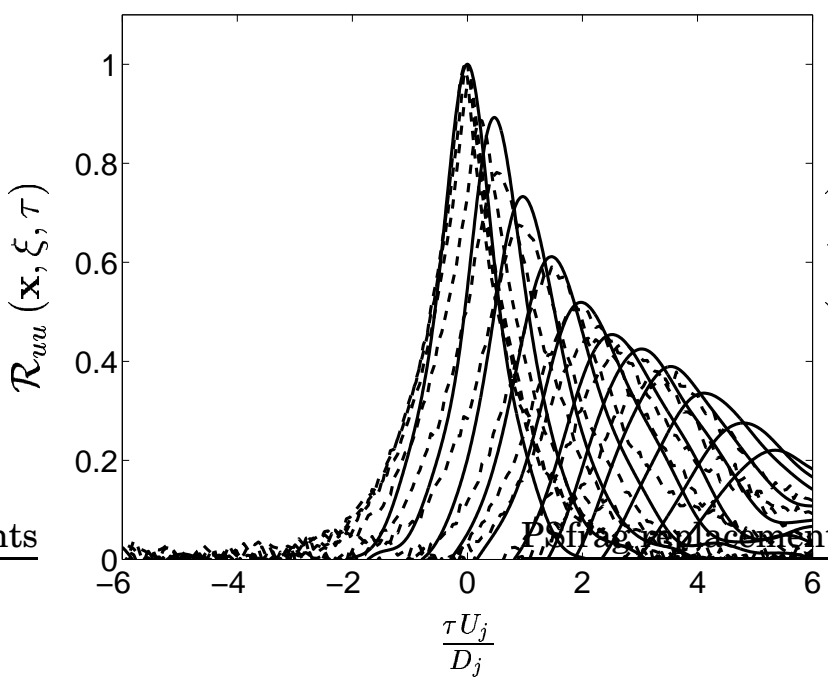

(a) Jet I

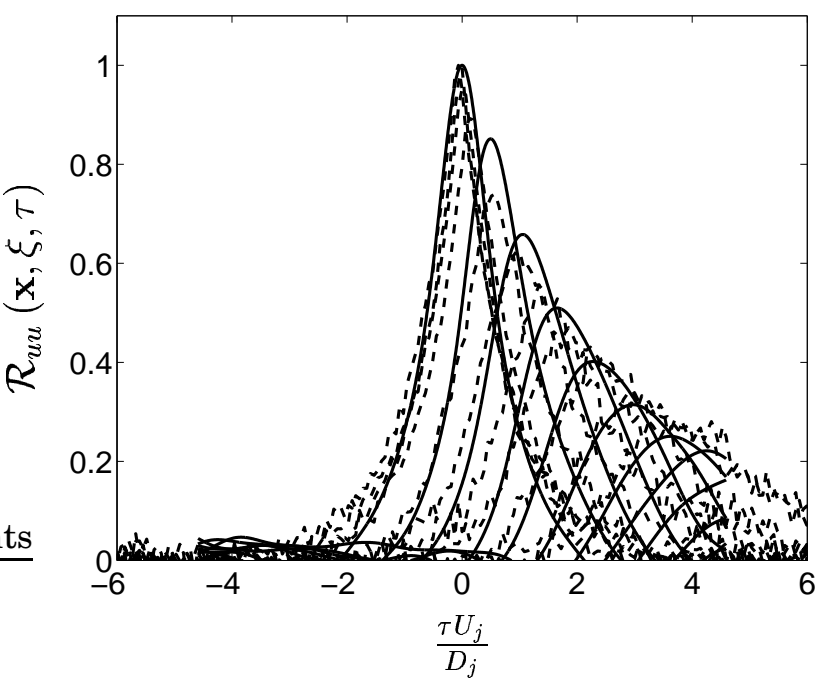

(b) Jet II

Figure 14. Two-point space-time correlations obtained in the shear layer at the end of the potential core, $\left(x=L_{c}, r=0.5 D_{j}\right)$. Solid lines denotes LES data and dashed lines measurements ${ }^{20, b}$.

Figure (16) gives a comparison of predicted correlations for Jet I and Jet II. The correlations are presented in the shear layer at axial positions normalized with the length of the potential core. As can be seen there are no great differences in the correlations for the two jets. However, since the potential core is shorter for Jet II, turbulence scales grow faster for this jet.

\section{Length and Time Scales of Turbulence Structures}

Estimates of local integral length and time scales of turbulence structures were obtained by integration of the corresponding autocorrelation and spatial correlation, respectively. The first crossing of the

\footnotetext{
${ }^{\mathrm{b}}$ P. Jordan, Private communication, 2003
} 


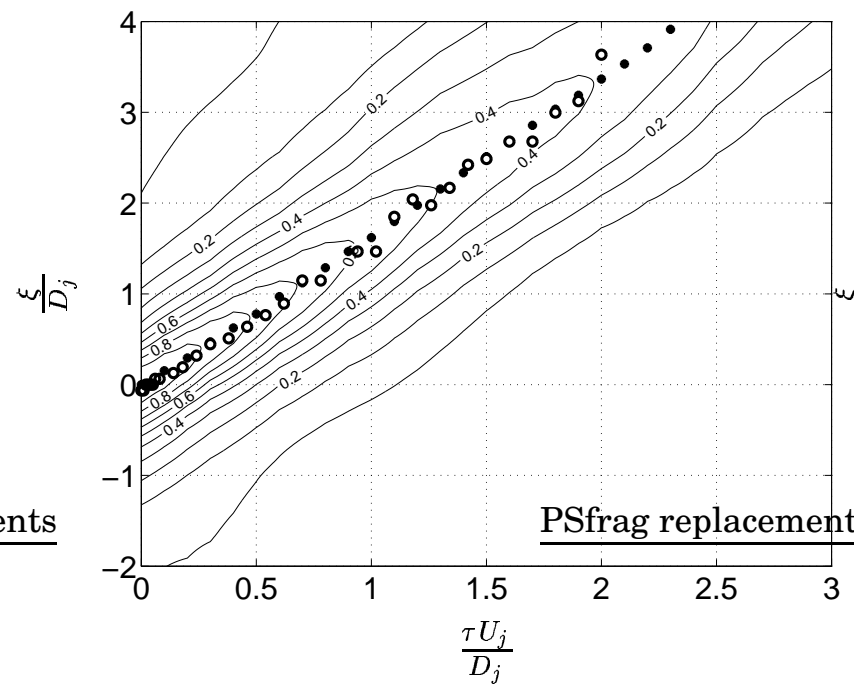

(a) Jet I

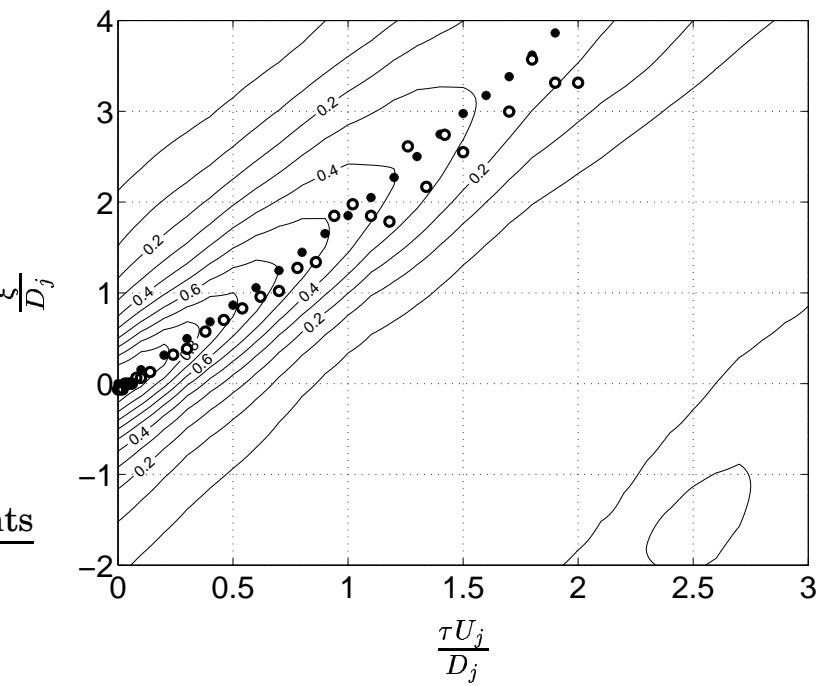

(b) Jet II

Figure 15. Contours of two-point space-time correlations obtained in the shear layer at the end of the potential core, $\left(x=L_{c}, r=0.5 D_{j}\right)$. The peaks of the predicted correlations are indicated by stars and the peaks of the measured data ${ }^{20, b}$ are indicated by circles.

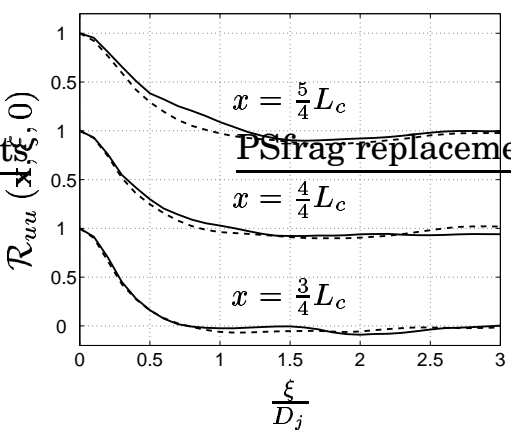

(a) Spatial correlations

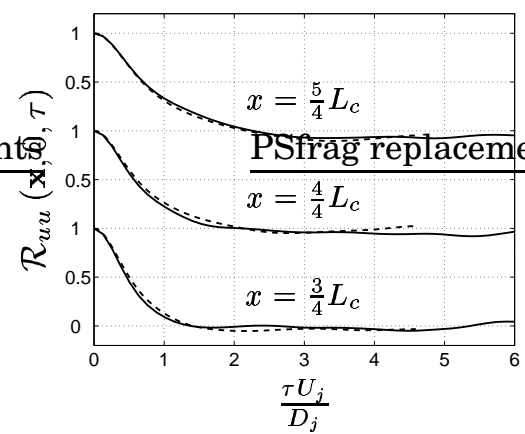

(b) Auto correlations

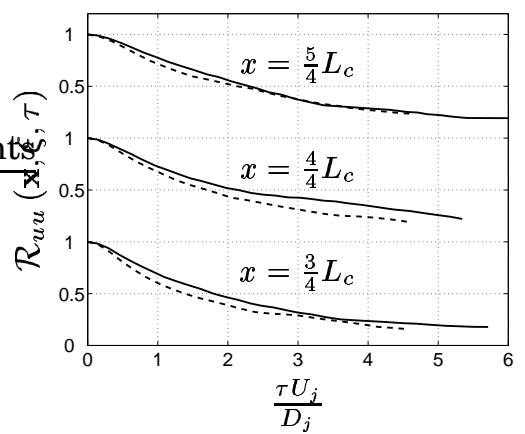

(c) Correlation envelopes

Figure 16. Comparison of spatial correlations, auto correlations and space-time correlation envelope for Jet I (solid lines) and Jet II (dashed lines).

coordinate axis was used as the upper limit for the integration. Figure (17) gives a comparison of length and time scales for Jet I and Jet II. In figure (17(a)), the axial development of predicted integral length scale is depicted. Note that to be able to make a comparison of the two jets, the axial coordinate has been normalized by the length of the potential core. Figure (17(b)) shows the axial development 
of integral time scale. Scales obtained from the two-point measurements ${ }^{\mathrm{c}}$ are indicated by circles and stars for Jet I and Jet II, respectively.

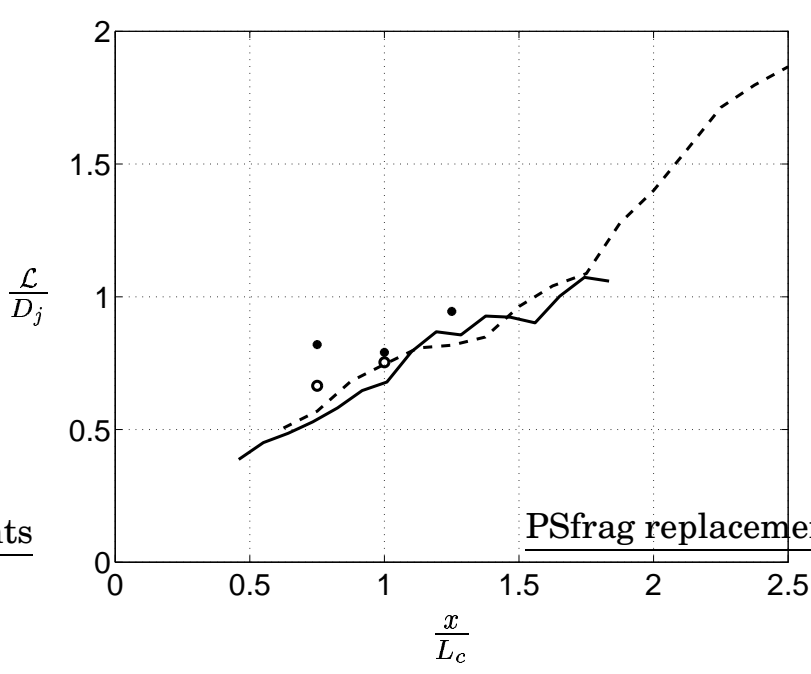

(a) Integral length scale $(\mathcal{L})$

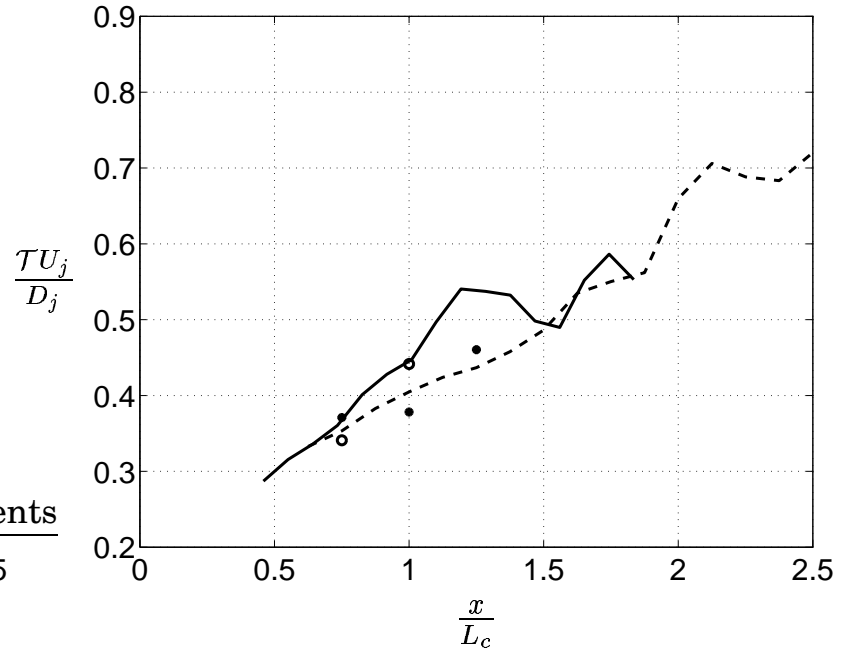

(b) Integral time scale $(\mathcal{T})$

Figure 17. Axial development of integral length and time scale. Solid line denotes Jet I and dashed line Jet II. circles and squares denotes the corresponding measured ${ }^{20, c}$ quantities.

\section{Far-field Sound Pressure Levels}

Power spectra of pressure fluctuations and sound pressure levels have been obtained for a few far-field observer locations. The observer locations coincide with the microphones in the experimental setup, ${ }^{19}$ see figure (18). The sound pressure level is here defined as

$$
S P L=20 \log _{10}\left(\frac{\sqrt{\left\langle\left(p^{\prime}\right)^{2}\right\rangle_{t}}}{p_{\text {ref }}}\right)
$$

where

$$
p_{\text {ref }}=\sqrt{\rho_{\infty} c_{\infty} 10^{-12}}=2.0 \times 10^{-5}[P a]
$$

Figure (19) shows a comparison of predicted and measured ${ }^{19}$ power spectra for a few observer locations on the inner arc, $\left(r=30 D_{j}\right)$. In order to make the comparison with measured data easier, the spectra have been filtered in third octave bands. It should be noted that the spectra have been staggered to be able to show them in the same graph. For low and high angles, the predicted levels are in good agreement with the experimental data up to at least Strouhal number $S t=1.5$, see figure (19). For the intermediate angles on the other hand, the predicted amplitude decreases rapidly above $S t=$ 1.0. This is due to the fact that the grid is not fine enough to support high frequency acoustic waves in the radial direction. Assuming that it is sufficient to have four cells per wavelength to be able to

\footnotetext{
${ }^{\mathrm{c}}$ P. Jordan, Private communication, 2003
} 
Far-field observers

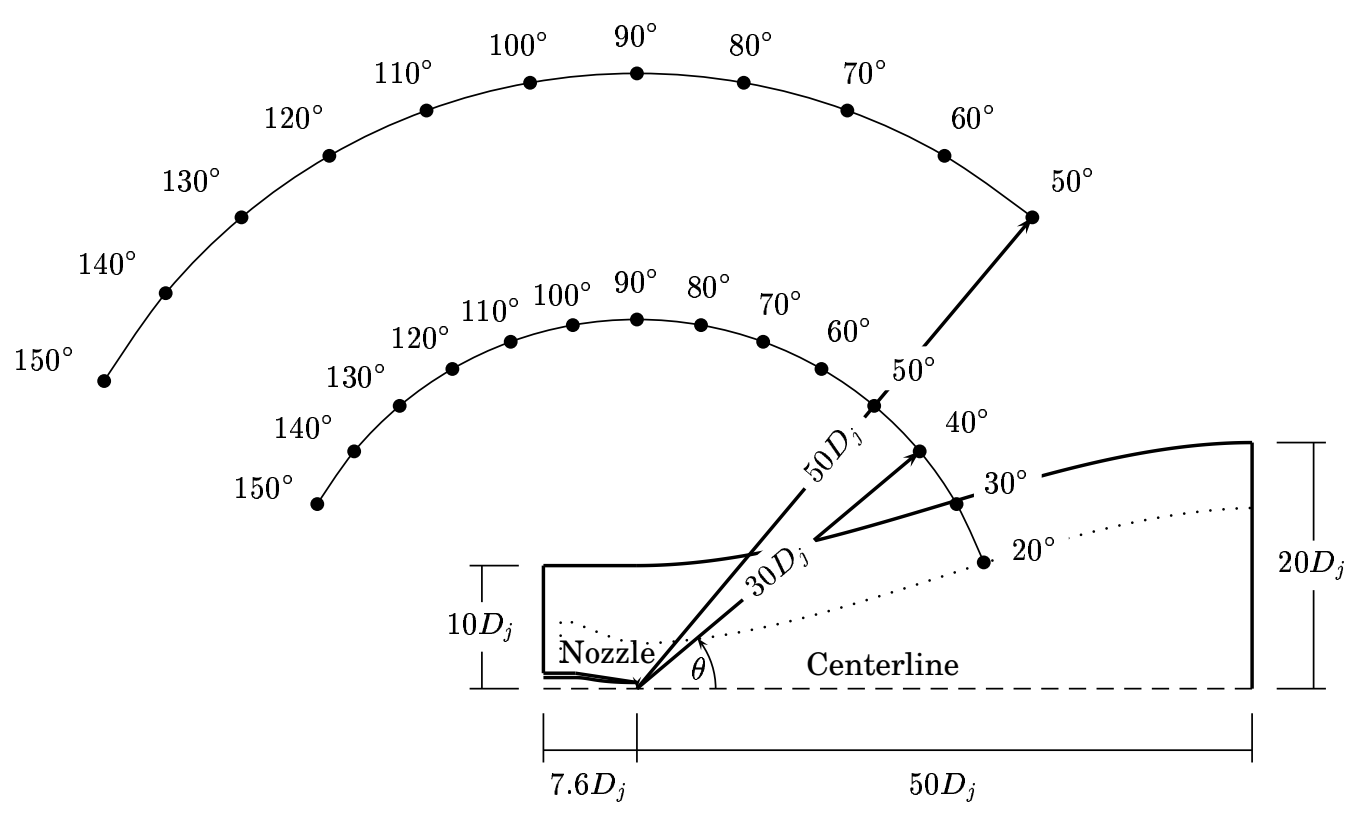

Figure 18. Acoustic pressure fluctuations were evaluated in 25 observer locations situated on two arcs in the far-field regions of the jet. The dotted line denotes the Kirchhoff surface.

capture a propagating wave of a certain frequency, the cells in the area where the Kirchhoff surface is located would support propagating waves of Strouhal numbers up to $S t \simeq 1.2$. This means that the mesh itself acts as a low-pass filter as the acoustic waves propagate through the domain. This effect is especially noticeable for the intermediate angles, where the mesh, due to the resolution demands at the jet exit, is very coarse, see figure (3(a)). The filtering of acoustic waves caused by the coarseness of the mesh might be an explanation for the somewhat overpredicted spectrum amplitudes in the high frequency range for the observers on the forward $\operatorname{arc}\left(\theta>90^{\circ}\right)$, see figure (19). This overprediction might be caused by unphysical, high frequency acoustic waves generated in the hydrodynamic jet region. An indication of such pollution of high frequencies by the presence of spurious noise was found when Lighthill's acoustic analogy was used for sound propagation, which has been reported in Andersson. ${ }^{7}$ These high frequency waves will, for most observers, be damped out by numerical dissipation. However, in the upstream direction, the resolution is significantly finer relative to the downstream direction, which means that some of these non-physical waves might reach the Kirchhoff surface and thus contribute to the predicted pressure signal. Figure (20) gives a comparison of predicted and measured SPL for the inner arc. The predicted SPL have been obtained by integration of the corresponding spectra excluding the contribution from frequencies lower than Strouhal number $S t=0.05$. The underprediction of the spectrum amplitude above $S t=1.0$, gives underpredicted SPL for the observers between $50^{\circ}$ and $120^{\circ}$ for Jet I. This effect does not show up in the sound pressure levels for Jet II though. For Jet II SPL for the $20^{\circ}$ observer is underpredicted, which also can be seen in the power spectrum for this observer location where the peak level is underpredicted. As can be seen in figure (18) the $20^{\circ}$ observer is very close to the integration surface, which may affect the results. 


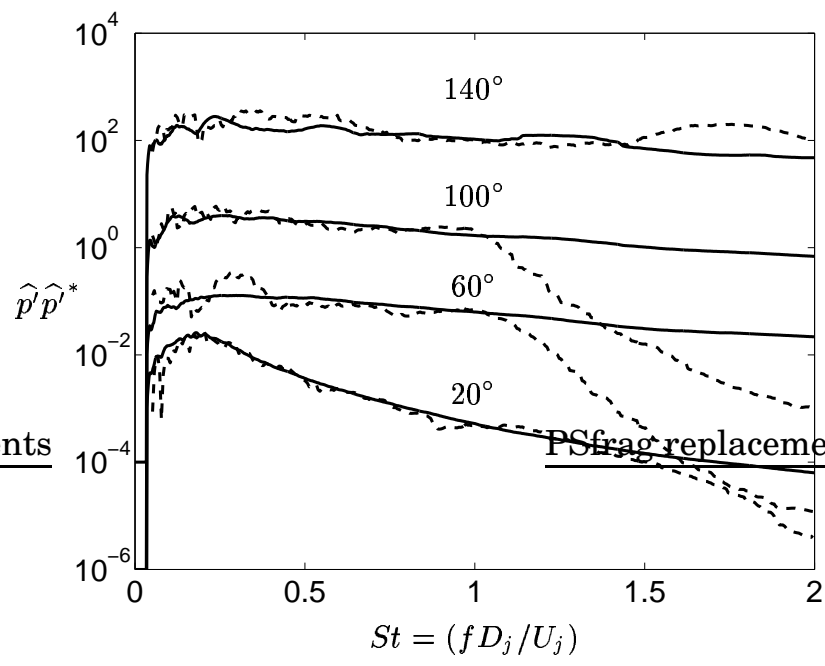

(a) Jet $I$

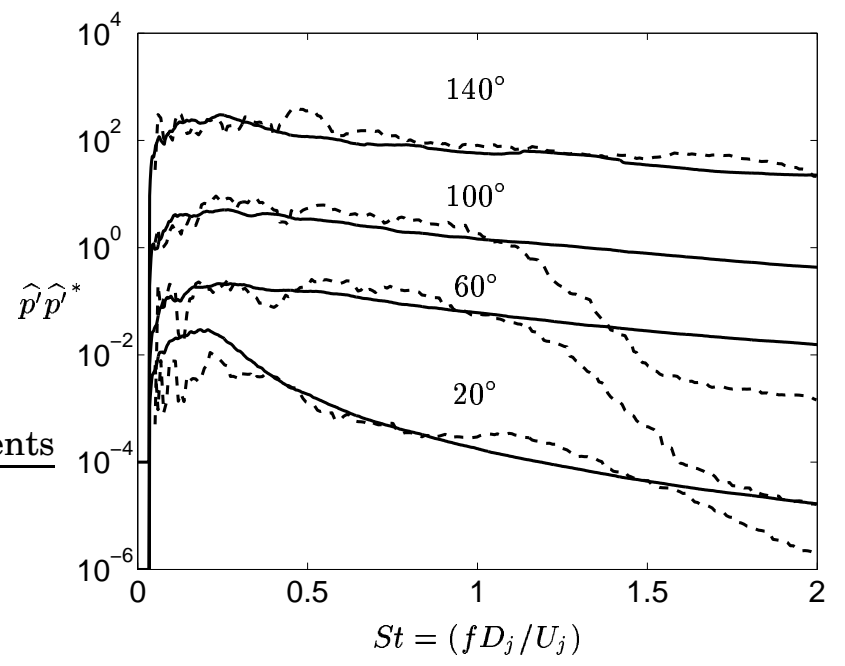

(b) Jet II

Figure 19. Power spectra of far-field pressure signal for a few observer locations on the inner $\operatorname{arc}\left(r=30 D_{j}\right)$. The spectra have been staggered by multiplying the amplitude by a factor $10^{2 n}$, where $n=\left(\frac{\theta-20}{40}\right), \theta$ being the angle from the jet axis, see figure (18). $\widehat{p^{\prime}}$ denotes the Fourier transform of the pressure fluctuation and $\widehat{p}^{*}$ its conjugate.

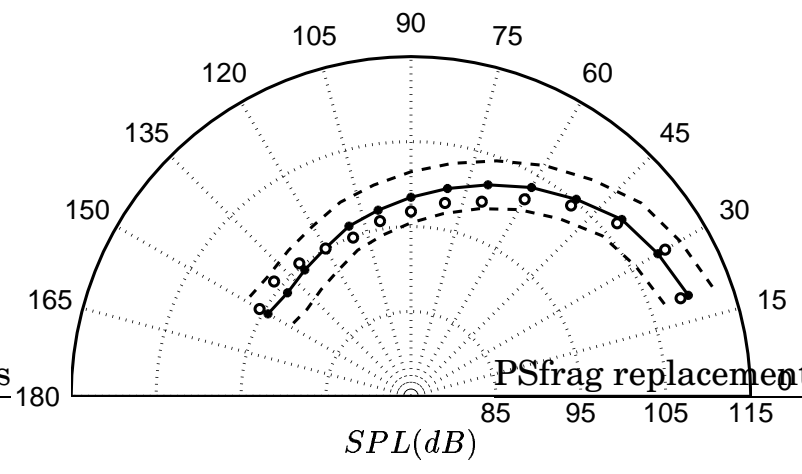

(a) Jet I

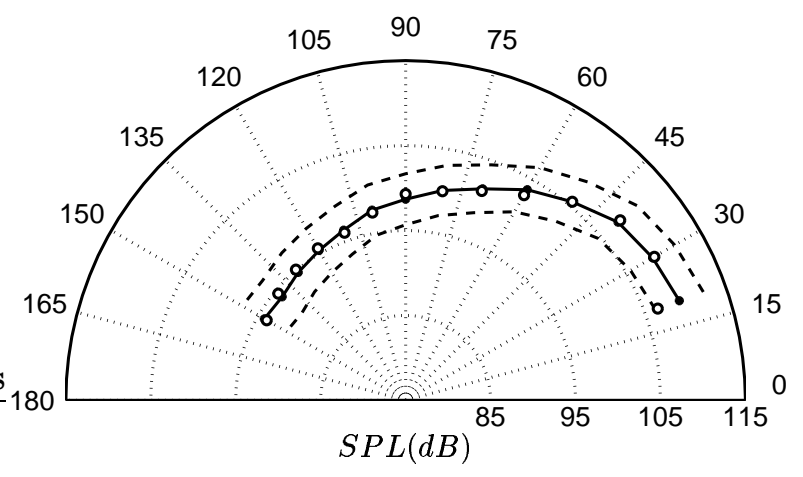

(b) Jet II

Figure 20. Sound pressure levels in observer locations situated $30 D_{j}$ from the nozzle outlet at different angles, $\theta$, measured from the jet axis, see figure (18). Solid line denotes measured levels and the dashed lines denotes measured levels $\pm 3.0[d B]$. Predicted SPL obtained using Kirchhoff surface integration are represented by circles.

Figures (21-22) show power spectra and sound pressure levels for the observers on the $50 D_{j}$ arc, respectively. 


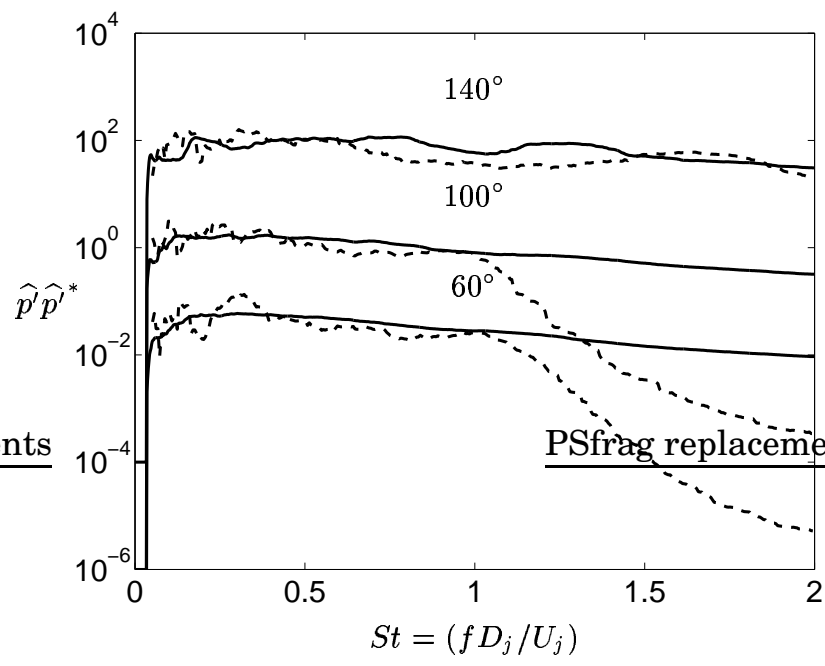

(a) Jet $I$

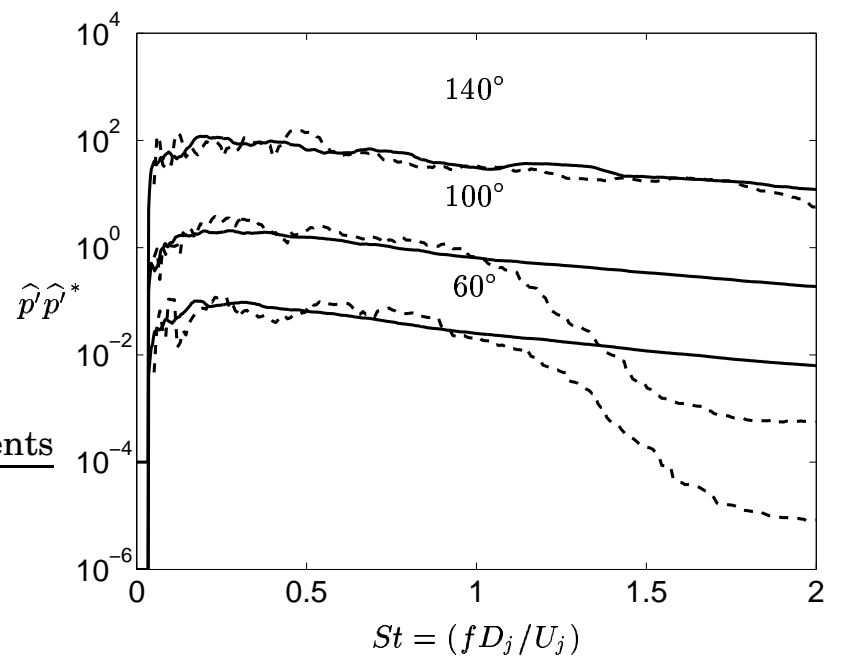

(b) Jet II

Figure 21. Power spectra of far-field pressure signal for a few observer locations on the outer arc $\left(r=50 D_{j}\right)$. See also legend to figure (19).

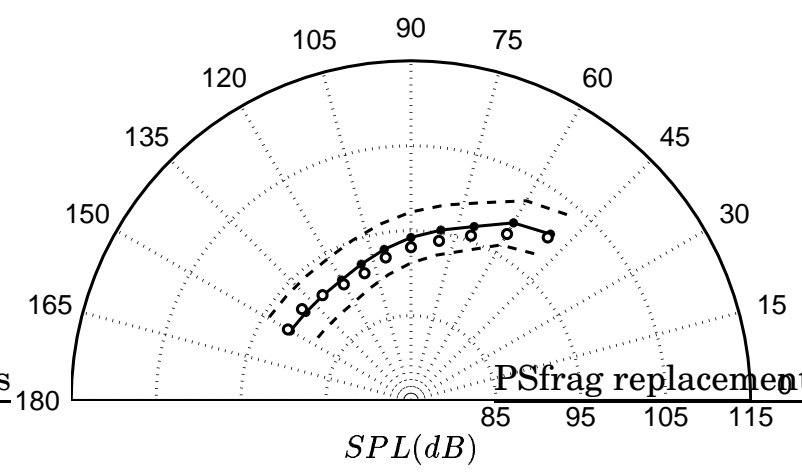

(a) Jet I

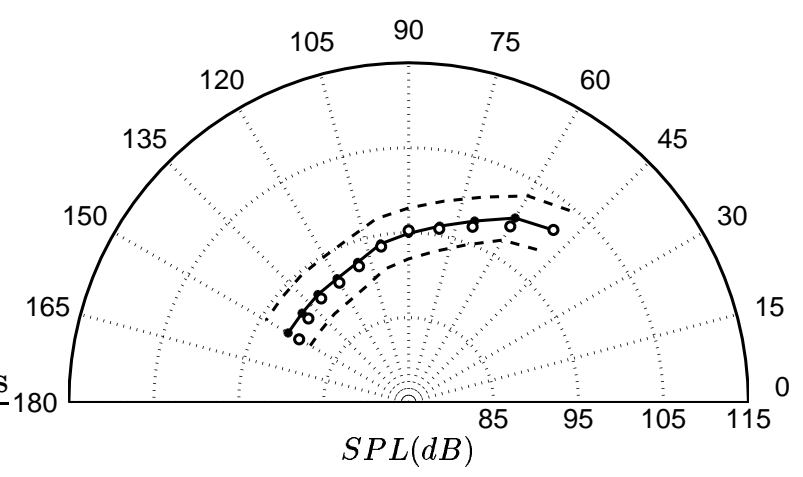

(b) Jet II

Figure 22. Sound pressure levels in observer locations situated $50 D_{j}$ from the nozzle outlet, see also legend to figure (20).

There are no great differences in the sound pressure levels obtained for Jet I and those obtained for Jet II. The levels for the heated jet is, however, slightly higher than for the cold jet, see figure (23). According to Jiang et al., ${ }^{30}$ increasing the jet to ambient temperature ratio results in a weaker sound source but increased sound pressure levels. Tests done to investigate the heating effects on the radiated sound have shown that for high exhaust speeds increasing the jet to ambient temperature ratio reduces the noise levels whereas at lower exhaust speeds the heated jet is the noisier, see Crighton. ${ }^{23}$ The slight increase in SPL found for Jet II is, however, not sufficiently large to be able to draw any conclusions 
regarding the heating effects on radiated sound in this study. Moreover, the measurements by Tanna, ${ }^{31}$ where a wide range of exhaust velocities and temperature ratios where tested, indicates that Mach number 0.75 is in the range where the trend changes from increase to decrease of emitted sound as increasing the temperature ratio, and thus the heating effects on the sound pressure levels in the far-field are small at this Mach number.

In summary, the predicted sound pressure levels are for all observers within the $3.0[d B]$ deviation from the measured levels indicated by dashed lines in figures (20) and (22), and for most of the observers within a $1.0[d B]$ deviation which must be considered as a satisfying result. It is important to note that these levels have been obtained directly from the LES/Kirchhoff data without adjustment.

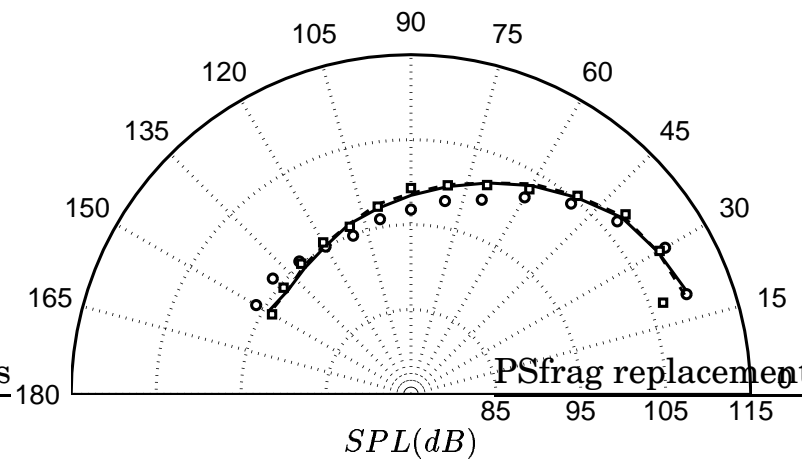

(a) $r=30 D_{j}$

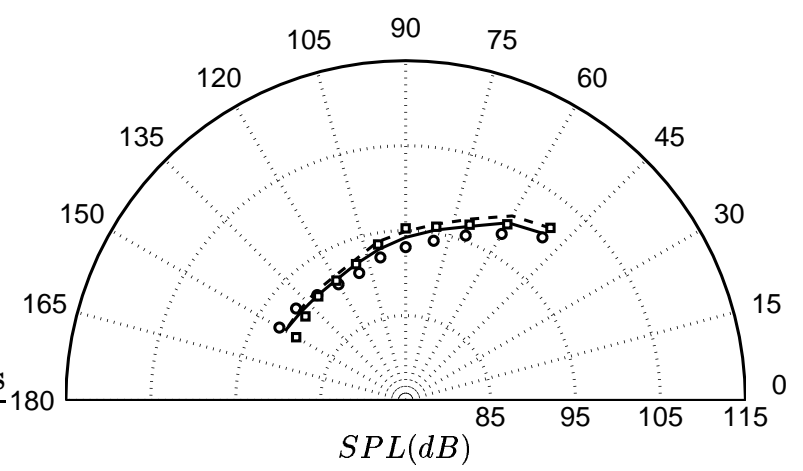

(b) $r=50 D_{j}$

Figure 23. Comparison of SPL for Jet I and Jet II. Solid lines and dashed lines denote measured data for Jet I and Jet II, respectively. Circles and squares denote predicted SPL.

\section{Concluding remarks}

LES of a compressible Mach 0.75 nozzle/jet configuration has been performed. The Reynolds number based on jet velocity and nozzle diameter was $5.0 \times 10^{4}$. Jet simulations were performed at two heating conditions, an unheated jet where the exhaust static temperature was equal to the static temperature of the surrounding air and a heated jet where the nozzle outlet temperature was twice that of the ambient air. Profiles of time and azimuthally averaged flow properties have been compared with data measured by Jordan et al. ${ }^{18}$ Although some deviations occur, the results are in general in very good agreement with experiments. The good results are probably attributed to the homogeneity of the mesh used and the fact that a nozzle geometry was included in the calculation domain. Using a combination of cartesian and polar mesh blocks makes it possible to ensure that cells in the outer regions of the calculation domain are not too large and prevent cells from being clustered in the centerline region.

The maximum levels of turbulence intensities are well captured for both jets. Furthermore, the turbulence anisotropy of the jets is correctly predicted. The initial jet spreading and the potential core lengths are however not predicted correctly. This might be due to the use of a grid too coarse in the nozzle outlet region, i.e. too coarse for the subgrid scale model used. Furthermore, the entrainment velocities specified at the free boundaries affect the jet spreading.

Two-point space-time correlations were obtained in a number of locations in the shear layer along the nozzle lip-line. Correlation curves obtained at $x=L_{c}$ were in good agreement with experiments. ${ }^{20}$ Two-point space-time correlations in the shear layer were used to obtain estimates of integral length 
scales, integral time scales and eddy convection velocities. The agreement with corresponding quantities obtained from the measured data was satisfying. Only small differences could be identified in correlations and integral scales for the two jets.

Sound pressure levels in the far-field were evaluated with a hybrid approach. Kirchhoff surface integration was utilized for the propagation of sound to far-field locations. Predicted sound pressure levels were in excellent agreement with the levels measured by Jordan et al. ${ }^{19}$ for both jets. However, the mesh used fails to capture waves of Strouhal numbers higher than $S t \simeq 1.2$. This results in a rapid fall-off of the spectrum amplitudes for higher Strouhal numbers. The lower part of the spectra is accurately captured, however. Since the sound pressure levels obtained were in good agreement with the measured levels, capturing the lower part of the spectra seems to be sufficient to represent the main part of the radiated sound. Both measurements and predictions show slightly higher SPL for the heated jet. The increase in SPL is, however, too small to be able to draw any conclusions regarding the effects of the increased temperature ratio on the radiated sound.

With a surface integral approach, it is sufficient to use a mesh resolving the main noise generation structures and supporting acoustic waves within the frequency range contributing most to the overall sound pressure levels. However, if the aim were to capture the sound spectra in far-field locations, higher mesh resolution and probably higher-order schemes would be desirable.

It is worth mentioning that the results presented in this paper have been obtained in spite of using a less accurate numerical scheme and a mesh of lower resolution than usually recommended for aeroacoustic applications.

\section{Acknowledgment}

This work was conducted as part of the EU 5th Framework Project JEAN (Jet Exhaust Aerodynamics \& Noise), contract number G4RD-CT2000-000313.

\section{References}

${ }^{1}$ Freund, J., "Noise Sources in a Low-Reynolds-number Turbulent Jet at Mach 0.9,” Journal of Fluid Mechanics, Vol. 438, 2001, pp. 277-305.

${ }^{2}$ Mitchell, B., Lele, S., and Moin, P., "Direct Computation of the Sound Generated by Vortex Pairing in an Axisymmetric Jet," Journal of Fluid Mechanics, Vol. 383, 1999, pp. 113-142.

${ }^{3}$ Bogey, C., Bailly, C., and Juvé, D., "Computation of the Sound Radiated by a 3-D Jet Using Large Eddy Simulation," The 6th AIAA / CEAS Aeroacoustics Conference, No. 2009 in AIAA 2000, Lahaina, Hawaii, 2000.

${ }^{4}$ Mankbadi, R., Shih, S., Hixon, R., and Povinelli, L., "Direct Computation of Jet Noise Produced by Large-Scale Axisymmetric Structures," J. Propulsion and Power, Vol. 16, No. 2, 2000, pp. 207-215.

${ }^{5}$ Mankbadi, R., "Review of Computational Aeroacoustics in Propulsion Systems," J. Propulsion and Power, Vol. 15, No. 4, 1999 , pp. 504-512.

${ }^{6}$ Andersson, N., Eriksson, L.-E., and Davidson, L., "Large-Eddy Simulation of a Mach 0.75 Jet," The 9th AIAA/CEAS Aeroacoustics Conference, No. 3312 in AIAA 2003, Hilton Head, South Carolina, 2003.

${ }^{7}$ Andersson, N., A Study of Mach 0.75 Jets and Their Radiated Sound Using Large-Eddy Simulation, Licentiate thesis, Division of Thermo and Fluid Dynamics, Chalmers University of Technology, Gothenburg, 2003.

${ }^{8}$ Bogey, C., Bailly, C., and Juvé, D., "Noise Investigation of a High Subsonic, Moderate Reynolds Number Jet Using a Compressible Large Eddy Simulation,” Theoret. Comput. Fluid Dynamics, Vol. 16, No. 4, 2003, pp. 273-297.

${ }^{9}$ Bogey, C., Bailly, C., and Juvé, D., "Noise Computation Using Lighthill's Equation with Inclusion of Mean Flow - Acoustic Interactions," The 7th AIAA/CEAS Aeroacoustics Conference, No. 2255 in AIAA 2001, Maastricht, Netherlands, 2001.

${ }^{10}$ Bogey, C. and Bailly, C., "LES of a High Reynolds, High Subsonic Jet : Effects of the Inflow Conditions on Flow and Noise." The 9th AIAA/CEAS Aeroacoustics Conference, No. 3170 in AIAA 2003, Hilton Head, South Carolina, 2003.

${ }^{11}$ Boersma, B., Brethouwer, G., and Nieuwstadt, F., "A Numerical Investigation on the Effect of the Inflow Conditions on the Self-similar Region of a Round Jet," Physics of Fluids, Vol. 10, No. 4, 1998, pp. 899-909.

${ }^{12}$ Freund, J., Lele, S., and Moin, P., "Numerical Simulation of a Mach 1.92 Jet and its Sound Field," AIAA Journal, Vol. 38, No. 11, 2000, pp. 2023-2031. 
${ }^{13}$ DeBonis, J. and Scott, J., "A Large-Eddy Simulation of a Turbulent Compressible Round Jet," The 7th AIAA/CEAS Aeroacoustics Conference, No. 2254 in AIAA 2001, Maastricht, Netherlands, 2001.

${ }^{14}$ Shur, M., Spalart, P., Strelets, M., and Travin, A., "Towards the Prediction of Noise from Jet Engines," International Journal of Heat and Fluid Flow, Vol. 24, No. 4, 2003, pp. 551-561.

${ }^{15}$ Le Ribault, C., Sarkar, S., and Stanley, S., "Large Eddy Simulation of a Plane Jet," Physics of Fluids, Vol. 11, No. 10, 1999, pp. 3069-3083.

${ }^{16}$ Zhao, W., Frankel, S., and Mongeau, L., "Large Eddy Simulation of Sound Radiation from Subsonic Turbulent Jets," AIAA Journal, Vol. 39, No. 8, 2001, pp. 1469-1477.

${ }^{17}$ Rembold, B., Adams, N., and Kleiser, L., "Direct Numerical Simulation of a Transitional Rectangular Jet," International Journal of Heat and Fluid Flow, Vol. 23, No. 5, 2002, pp. 547-553.

${ }^{18}$ Jordan, P., Gervais, Y., Valiére, J.-C., and Foulon, H., "Final Results from Single Point Measurements," Project deliverable D3.4, JEAN - EU 5th Framework Programme, G4RD-CT2000-00313, Laboratoire d'Etude Aeródynamiques, Poitiers, 2002.

${ }^{19}$ Jordan, P., Gervais, Y., Valiére, J.-C., and Foulon, H., "Results from Acoustic Field Measurements," Project deliverable D3.6, JEAN - EU 5th Framework Programme, G4RD-CT2000-00313, Laboratoire d'Etude Aeródynamiques, Poitiers, 2002.

${ }^{20}$ Jordan, P. and Gervais, Y., "Modeling Self and Shear Noise Mechanisms in Anisotropic Turbulence," The 9th AIAA/CEAS Aeroacoustics Conference, No. 8743 in AIAA 2003, Hilton Head, South Carolina, 2003.

${ }^{21}$ Erlebacher, G., Hussaini, M., Speziale, C., and Zang, T., "Toward the Large-Eddy Simulation of Compressible Turbulent Flows," Journal of Fluid Mechanics, Vol. 238, 1992, pp. 155-185.

${ }^{22}$ Lighthill, M., "On Sound Generated Aerodynamically, I. General Theory," Proc. Roy. Soc., Vol. A 211, 1952, pp. 564-587.

${ }^{23}$ Crighton, D., "Basic Principles of Aerodynamic Noise Generation," Progress in Aerospace Sciences, Vol. 16 No. 1, 1975, pp. 31-96.

${ }^{24}$ Lyrintzis, A., "Review: The Use of Kirchhoff's Method in Computational Aeroacoustics," ASME: Journal of Fluids Engineering, Vol. 116, 1994, pp. 665-676.

${ }^{25}$ Freund, J., Lele, S., and Moin, P., "Calculation of the Radiated Sound Field Using an Open Kirchhoff Surface," AIAA Journal, Vol. 34, No. 5, 1996, pp. 909-916.

${ }^{26}$ Rahier, G., Prieur, J., Vuillot, F., Lupoglazoff, N., and Biancherin, A., "Investigation of Integral Surface Formulations for Acoustic Predictions of Hot Jets Starting from Unsteady Aerodynamic Simulations," The 9th AIAA/CEAS Aeroacoustics Conference, No. 3164 in AIAA 2003, Hilton Head, South Carolina, 2003.

${ }^{27}$ Ffowcs Williams, J. and Hawkings, D., "Sound Generated by Turbulence and Surfaces in Arbitrary Motion," Phil. Trans. Roy. Soc., Vol. A 264, 1969, pp. 321-342.

${ }^{28}$ Eriksson, L.-E., "Development and Validation of Highly Modular Flow Solver Versions in G2DFLOW and G3DFLOW," Internal report 9970-1162, Volvo Aero Corporation, Sweden, 1995.

${ }^{29}$ Hinze, J., Turbulence, McGraw Hill, 2nd ed., 1975, ISBN 0-07-029037-7.

${ }^{30}$ Jiang, X., Avital, E., and Luo, K., "Sound Generation by Vortex Pairing in Subsonic Axisymmetric Jets," AIAA Journal, Vol. 42, No. 2, 2004, pp. 241-248.

${ }^{31}$ Tanna, H., "An Experimental Study of Jet Noise Part I: Turbulent Mixing Noise," Journal of Sound and Vibration, Vol. 50, No. 3,1977 , pp. $405-428$. 